\title{
Der internationale Anwendungsbereich der Geschlechterquote für Großunternehmen
}

\author{
Prof. Dr. Marc-Philippe Weller, Wiss. Mit. Charlotte Harms, \\ Wiss. Mit. Bettina Rentsch \\ und \\ Akad. Rat Dr. Chris Thomale, LL.M. (Yale), Heidelberg*
}

Inhaltsübersicht

ZGR 2015, 361-395

I. Einführung . . . . . . . . . . . . . . . . . . . . . . . . . 362

II. Die Kernelemente des Gesetzes . . . . . . . . . . . . . . . . . . . . . . 364

III. Die Anwendbarkeit auf die Societas Europaea (SE) . . . . . . . . . . . . . . . . 366

1. Keine Regelungskompetenz des deutschen Gesetzgebers? . . . . . . . . . . 366

2. Quote als Bestandteil des sekundären Gesellschaftsstatuts (Art. 9 Abs. 1 lit. c SE-VO) . . . . . . . . . . . . . . . . . . . 367

3. Keine Sperrwirkung der SE-Mitbestimmungsrichtlinie (RL 2001/86/EG) 367

IV. Die Anwendbarkeit auf Auslandsgesellschaften - der kollisionsrechtliche Rahmen . . . . . . . . . . . . . . . . . . . . . . 369

V. Die Quotenregelung als Eingriffsnorm . . . . . . . . . . . . . . . . . 370

1. Begriff und Wirkungsweise von Eingriffsnormen . . . . . . . . . . . . . . . 370

2. Beispiele für Eingriffsnormen im Unternehmensrecht . . . . . . . . . . 371

3. Der eingriffsrechtliche Charakter der $\$ \mathbb{S} 96$ Abs. 2 AktG-Entwurf und 24 Abs. 3 SEAG-Entwurf . . . . . . . . . . . . . . . . . 374

VI. Sonderanknüpfung der Quotenregelung . . . . . . . . . . . . . . . . 378

1. Reichweite des internationalen Geltungswillens: Nur Quotenregelung, nicht Implementierungs- und Sanktionierungsregime . . . . . . . . . . . . 378

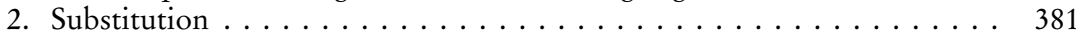

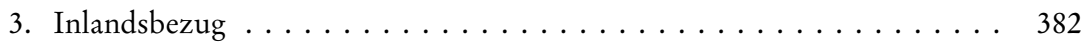

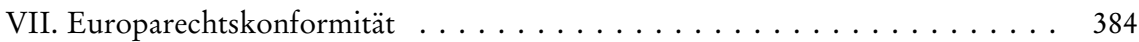

1. Gewährleistungsbereich der Niederlassungsfreiheit . . . . . . . . . . . . . . . 385

2. Beschränkung der Niederlassungsfreiheit . . . . . . . . . . . . . . 386

3. Keck-Ausnahme: Quotenregelung als bloße Tätigkeitsausübungsmodali-

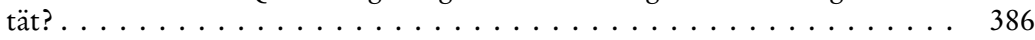

4. Zwingender Grund des Allgemeinwohls . . . . . . . . . . . . . . . . . 389

* Die Verf. forschen am Institut für ausländisches und internationales Privat- und Wirtschaftsrecht der Universität Heidelberg. Der Beitrag geht auf eine Vortragsveranstaltung des „Forums Gleichstellung“ an der Universität Heidelberg zurück. Der Erstautor hat im Jahr 2011 für das Bundesfamilienministerium ein Gutachten zur „Flexiquote“ erstellt. In Bezug auf die im Beitrag diskutierte starre 30\%-Geschlechterquote fungierte der Erstautor im Februar 2015 als Sachverständiger des Rechts- und Familienausschusses des Deutschen Bundestages. 
5. Geeignetheit der Quote: Kein Verstoß gegen Art. 157 Abs. 3, 4 AEUV . . 390

6. Erforderlichkeit und Angemessenheit . . . . . . . . . . . . . 393

VIII. Zusammenfassung in Thesenform . . . . . . . . . . . . . . . . . 394

Das von der Bundesregierung beschlossene Gesetz für die gleichberechtigte Teilhabe von Frauen und Männern an Führungspositionen in der Privatwirtschaft vom 06. 03. 2015 statuiert im Kern eine 30\%-Geschlechterquote für den Aufsichtsrat in der Aktiengesellschaft sowie für den Verwaltungsrat in der monistischen Societas Europaea. Der Beitrag beleuchtet den internationalen Anwendungsbereich der Quotenregelung und hält dafür, dass die 30\%-Regelauch auf Auslandsgesellschaften mit hinreichendem Inlandsbezug angewendet werden kann.

On 06 March 2015, the German Federal Government adopted a bill on equal participation of women and men in private sector executive positions. The bill establishes a mandatory $30 \%$ gender quota in the supervisory board of the Aktiengesellschaft (Public Limited Company) and in the board of directors of the Societas Europaea in case of a monistic governance system. This article discusses the quota's international and extraterritorial scope and advocates for its extensive application to foreign companies with significant contacts to the German Federal legal order.

\section{Einfübrung}

Die geplante Geschlechterquote in Großunternehmen sorgt für reichlichen Diskussionsstoff ${ }^{1}$. Das Kernelement des Gesetzes der Bundesregierung ${ }^{2}$ liegt in der Einführung einer 30\%-Quote für den Aufsichtsrat von Aktiengesellschaften, die börsennotiert und mitbestimmt sind (unter II.). Die Erstreckung der Quotenregelung auf die Societas Europaea ist umstritten (unter III.). Ebenso wenig ausgeleuchtet erscheint ferner bislang die Frage nach der internationalen Anwendbarkeit der geplanten Quotenregelung. Diese ist u. a. deshalb interessant, weil die Einführung einer 40\%-Quote im Jahr 2008 in Norwegen zu einer „Flucht“ aus der Rechtsform der Aktiengesellschaft geführt hat ${ }^{3}$. Eine ähnliche Rechtsarbitrage zur Umgehung gesellschaftspolitischer Normen ist auch in Deutschland nicht unbekannt: So versuchen einige Großunternehmen, dem MitbestG 1976 und dem DrittelbeteiligungsG 2004 auszuweichen, indem sie sich nach einem ausländischen oder unionsharmonisierten Gesellschafts-

1 Die geplante starre Quotenreglung wird teilweise sogar für verfassungswidrig gehalten, Habersack/Kersten, BB 2014, 2819, 2830, Thesen 4 und 5.

2 Der Bundestag hat am 6. März 2015 das Gesetz für die gleichberechtigte Teilhabe von Frauen und Männern an Führungspositionen in der Privatwirtschaft und im öffentlichen Dienst in der Fassung der Beschlussempfehlung des Ausschusses für Familie, Senioren, Frauen und Jugend verabschiedet, BT-Drucks. 18/92, S. 8761 C; zur Beschlussempfehlung vgl. BT-Drucks. 18/4227.

3 Ahern/Dittmar, The Changing of the Boards: The Impact on Firm Valuation of Mandated Female Board Representation, The Quarterly Journal of Economics (2012) 127 (1), 137 (183 ff). 
statut organisieren ${ }^{4}$. Als prominente Beispiele gelten etwa die Fluggesellschaft Air Berlin (organisiert als PLC), die Drogeriemarktkette Müller (Ltd. \& Co KG) sowie die Deutschland-Tochtergesellschaften des Landmaschinenherstellers John Deer und der Fastfood-Kette McDonald's, die jeweils als Incorporations (Inc.) US-amerikanischen Rechts ${ }^{5}$ organisiert sind ${ }^{6}$. Für die Geschlechterquote wird ebenfalls eine Fluchtbewegung prognostiziert ${ }^{7}$.

Das europäische Kollisionsrecht erlaubt im Anschluss an das Centros-Judikat des $\mathrm{EuGH}^{8}$ durchaus den Rekurs auf EU-Auslandsgesellschaften zur Umgehung inländischer gesellschaftsrechtlicher Standards (unter IV.). Allerdings wird das Internationale Gesellschaftsrecht durch das Eingriffsrecht begrenzt. Dabei handelt es sich um international zwingende Bestimmungen der lex fori, mithin der Rechtsordnung des Aufnahmestaats, die sich anerkanntermaßen gegen das ausländische Gesellschaftsstatut der Auslandsgesellschaft durchzusetzen vermögen, sofern ein hinreichender Bezug zum Inland besteht ${ }^{9}$. Beurteilt man die Geschlechterquotenregelung unter dem Gesichtspunkt dieser kollisionsrechtlichen Eingriffsnormendogmatik, ist die 30\%-Quotenvorgabe grundsätzlich als Eingriffsnorm zu qualifizieren (unter V.). Dies wirft freilich die Vorfragen auf, ob die Regelungen in $\$ 96$ Abs. 2 AktG-Entwurf und $\$ 24$ Abs. 3 SEAG-Entwurf einerseits überhaupt international gelten sollen bzw. inwieweit ihre Tatbestandsmerkmale „AG“ und „SE“ durch gleichwertige ausländische Rechtsformen substituiert werden können. Hier ergibt sich ein

4 Näher Weller, FS Hommelhoff, 2012, S. 1275 ff. Dass auch die SE sogar ganz überwiegend als „Vorratsgesellschaft“ zur Umgehung der unternehmerischen Mitbestimmung genutzt wird, belegt die empirische Studie von Schubert/von Der HöH, AG 2014, 439, 442 f. Diese Möglichkeit besteht wegen der Ausgestaltung der SE als nicht originär mitbestimmte Rechtsform, die nach der Ergänzungsrichtlinie 2001/86/EG vom 8. Oktober 2001 sowie nach deutschem $\$ \mathbb{S} 18$ Abs. 3, 34 ff SEBG grundsätzlich das alte Mitbestimmungsniveau auch dann „konserviert“, wenn die Schwellen von 500 Arbeitnehmern für das Drittelbeteiligungsgesetz und 2000 Arbeitnehmern für das Mitbestimmungsgesetz in der Folge überschritten werden. Siehe dazu Riesenhuber, Europäisches Arbeitsrecht, 2009, \29 Rdn.7, 46; Grundmann, Europäisches Gesellschaftsrecht, 2. Aufl., 2011, $\$ 29$ Rdn. 1089.

5 Zur Incorporation MERKT, US-amerikanisches Gesellschaftsrecht, 3. Aufl., 2014, S. $184 \mathrm{ff}$.

6 SICK, Mitbestimmungsrelevante Unternehmen mit ausländischen Rechtsformen, Studie der Hans Böckler-Stiftung, 2010, S. 5; DERs., GmbHR 2011, $1196 \mathrm{ff}$.

7 Tödtmann, Teure Flucht vor der Frauenquote, Wirtschaftswoche v. 24. 6. 2014. In dem Artikel gemeint ist die Flucht in die SE, weil diese nach dem ursprünglichen Referentenentwurf nicht von der Quotenregelung erfasst sein sollte; aber der Gedanke passt - wie bei der Mitbestimmung - auch in Bezug auf die Flucht in Auslandsgesellschaften. Vgl. auch PüTz/Weckes, Geschlechterquote, Report Nr. 1-2014 der Hans Böckler-Stiftung, 2014, S. 11.

8 EuGH, Urt. v. 9.3.1999, Rs. C-212/97, Slg. 1999, I-1459 - Centros.

9 Zur Sonderanknüpfung von Eingriffsnormen im Gesellschaftsrecht WeLler, Münchener Komm. z. GmbHG, 2. Aufl., 2015, Einleitung Int. GesR Rdn. 426 ff, 444 ff. 
differenziertes Bild: Während sich die 30\%-Quotenvorgabe als solche auch gegen ein ausländisches Gesellschaftsstatut durchsetzt, sind das Implementierungs- und das Sanktionierungsregime der $\$ 96$ Abs. 2 AktG-Entwurf und $\$ 24$ Abs. 3 SEAG-Entwurf, d.h. die Art und Weise der Aufsichtsratsbestellung sowie die Nichtigkeitsfolge im Fall quotenwidriger Besetzung auf Auslandsgesellschaften nicht anzuwenden (unter VI.). Die in der Sonderanknüpfung der Quotenregelung liegende Beschränkung der Niederlassungsfreiheit (Art. 49, 54 AEUV) ist unionsrechtskonform, weil sie entweder nach den verallgemeinerungsfähigen Grundsätzen der Keck-Rechtsprechung des $\mathrm{EuGH}^{10}$ als Bereichsausnahme vom sachlichen Schutzbereich der Niederlassungsfreiheit aufrecht erhalten oder jedenfalls nach der Cassis-Formel gerechtfertigt werden kann. Von Bedeutung ist dabei, dass sich die Einführung einer inhaltlich starren Quotenregelung als spezifische Besserstellung nach Art. 157 Abs. 4 AEUV legitimieren lässt (unter VII.).

\section{Die Kernelemente des Gesetzes}

Das Gesetz für die gleichberechtigte Teilhabe von Frauen und Männern an Führungspositionen in der Privatwirtschaft und im öffentlichen Dienst soll den geringen Anteil weiblicher Führungskräfte in der Privatwirtschaft und Bundesverwaltung erhöhen ${ }^{11}$. Für die Bundesverwaltung soll eine 30\%-Quote für Aufsichtsratsgremien im Einflussbereich des Bundes gelten, die ab 2018 auf $50 \%$ erhöht werden soll ${ }^{12}$. In Bezug auf die Privatwirtschaft muss sich der Vorstand mitbestimmter oder börsennotierter Gesellschaften für die beiden Führungsebenen, die unterhalb des Vorstandes angesiedelt sind, selbst definierte Zielgrößen zur Erhöhung des Frauenanteils setzen, deren (Nicht-)Einhaltung dokumentiert und begründet werden muss, die im Übrigen aber nicht durch scharfe Sanktionen bewehrt ist ${ }^{13}$. Dieselbe Pflicht trifft auch den Aufsichtsrat hinsichtlich des Vorstandes und sich selbst ${ }^{14}$. Eine bereits erreichte Zielgröße darf bis zur Erreichung einer 30\%-Quote nicht durch das Setzen einer geringeren Zielgröße unterschritten werden (Verschlechterungsverbot $)^{15}$.

10 Zur Übertragung der Keck-Rechtsprechung betreffend die Warenverkehrsfreiheit auf die Niederlassungsfreiheit Weller, Europäische Rechtsformwahlfreiheit und Gesellschafterhaftung, 2004, S. $34 \mathrm{ff}$.

11 BT-Drucks. 18/3784, S. 1 ff.

12 BT-Drucks. 18/3784, S. 7 f, Art. $1 \rrbracket \mathbb{\$} 1$, 4 Abs. 1 u. 2 BGremBG-E.

13 BT-Drucks. 18/3784, S.29, 37, Art. 3 \$76 Abs. 4 AktG-E, Art. 12, \289 a Abs. 2 Nr. 4 HGB-E.

14 BT-Drucks. 18/3784, S. 29, 37, Art. 3 \$111 Abs. 5 AktG-E, Art. $12 \rrbracket 289$ a Abs. 2 Nr. 4 HGB-E.

15 BT-Drucks. 18/3784, S. 29 f, Art. 3 \$76 Abs. 4 Satz 2, 3 AktG-E, $\mathbb{1 1 1}$ Abs. 5 Satz 2, 3 AktG-E. 
Kernelement des Quotengesetzes ist die starre Geschlechterquotenregelung für den Aufsichtsrat. Die 30\%-Mindestquote für Aufsichtsräte soll nach $\$ 96$ Abs. 2 AktG-Entwurf für sowohl paritätisch mitbestimmte als auch börsennotierte Gesellschaften greifen. Sie würde derzeit 108 Großunternehmen in Deutschland betreffen ${ }^{16}$. Die Quote ist grundsätzlich vom gesamten Aufsichtsrat zu erfüllen (Gesamterfüllung ${ }^{17}$. Hiernach kann eine höhere Quote auf Anteilseigner- oder Arbeitsnehmerseite ein entsprechendes Defizit der jeweils anderen Seite ausgleichen. Ausnahmsweise ist die 30\%-Quote für die Anteilseigner- und die Arbeitnehmerseite jedoch getrennt zu beachten, wenn die Seite der Anteilseigner- oder Arbeitnehmervertreter vor der Wahl einer Gesamterfüllung widerspricht (Getrennterfüllung) ${ }^{18}$. Die Nichtbeachtung der starren Quotenregelung wird mit der Nichtigkeit der Aufsichtsratswahl sanktioniert ${ }^{19}$. Das Gesetz v. 06.03.2015 fügt in der Fassung der Beschlussempfehlung v. 04.03.2015 dementsprechend nach dem geltenden Absatz 1 des $\$ 96$ AktG zur Zusammensetzung des Aufsichtsrats folgende Bestimmung als neuen Absatz 2 ein:

„Bei börsennotierten Gesellschaften, für die das Mitbestimmungsgesetz, das Montan-Mitbestimmungsgesetz oder das Mitbestimmungsergänzungsgesetz gilt, setzt sich der Aufsichtsrat zu mindestens 30 Prozent aus Frauen und zu mindestens 30 Prozent aus Männern zusammen. Der Mindestanteil ist vom Aufsichtsrat insgesamt zu erfüllen. Widerspricht die Seite der Anteilseigneroder Arbeitnehmervertreter auf Grund eines mit Mehrheit gefassten Beschlusses vor der Wahl der Gesamterfüllung gegenüber dem Aufsichtsratsvorsitzenden, so ist der Mindestanteil für diese Wahl von der Seite der Anteilseigner und der Seite der Arbeitnehmer getrennt zu erfüllen. Es ist in allen Fällen auf volle Personenzahlen mathematisch auf- beziehungsweise abzurunden. Verringert sich bei Gesamterfüllung der höhere Frauenanteil einer Seite nachträglich und widerspricht sie nun der Gesamterfüllung, so wird dadurch die Besetzung auf der anderen Seite nicht unwirksam. Eine Wahl der Mitglieder des Aufsichtsrats durch die Hauptversammlung und eine Entsendung in den Aufsichtsrat unter Verstoß gegen das Mindestanteilsgebot ist nichtig. Ist eine Wahl aus anderen Gründen für nichtig erklärt, so verstoßen zwischenzeitlich erfolgte Wahlen insoweit nicht gegen das Mindestanteilsgebot. Auf die Wahl der Aufsichtsratsmitglieder der Arbeitnehmer sind die in Satz 1 genannten Gesetze zur Mitbestimmung anzuwenden.“

Eine 30\%-Quote soll nach $\$ 17$ Abs. 2 SEAG-Entwurf auch für das Aufsichtsorgan einer dualistischen Societas Europaea (SE) sowie nach $\$ 24$ Abs. 3 SEAG-Entwurf für den Verwaltungsrat einer monistischen SE gelten, sofern diese aufgrund einer Vereinbarung oder bei Anwendung der Auffangregelung nach dem SE-Beteiligungsgesetz (SEBG) paritätisch mitbestimmt sind ${ }^{20}$. In Bezug auf die monistische SE soll dem $\$ 24$ SEAG ein dritter Absatz angefügt werden:

16 Auflistung der Unternehmen bei Pütz/Weckes, Geschlechterquote, Report Nr. 1-2014 der Hans Böckler-Stiftung, 2014, S. 5.

17 BT-Drucks. 18/3784, S. 29 f, Art. 3 \$96 Abs. 2 Satz 2 AktG-E.

18 BT-Drucks. 18/3784, S. 29 f, Art. 3 \$ 96 Abs. 2 Satz 3 AktG-E.

19 BT-Drucks. 18/3784, S. 29 f, Art. 3 \$96 Abs. 2 Satz 4 AktG-E.

20 BT-Drucks. 18/3784, S. 38, Art. 14 \$S 17 Abs. 2; 24 Abs. 3 SEAG-E. 
„Besteht bei einer börsennotierten SE der Verwaltungsrat aus derselben Zahl von Anteilseignerund Arbeitnehmervertretern, müssen in dem Verwaltungsrat Frauen und Männer jeweils mit einem Anteil von mindestens 30 Prozent vertreten sein (...).“

Die Nichtigkeitssanktion bei quotenwidriger Bestellung des Aufsichtsorgans der SE soll sich laut Gesetz aus der SE-VO selbst ergeben ${ }^{21}$.

\section{Die Anwendbarkeit auf die Societas Europaea (SE)}

\section{Keine Regelungskompetenz des deutschen Gesetzgebers?}

In der Literatur wird vertreten, für die SE könne keine starre Geschlechterquote für den Aufsichtsrat eingeführt werden ${ }^{22}$. Dem Bundestag fehle zumindest hinsichtlich der Quote auf Arbeitnehmerseite aufgrund der SE-Mitbestimmungsrichtlinie (RL 2001/86/EG) die Regelungskompetenz; nur der europäische Gesetzgeber könne durch Änderung der Richtlinie eine Quote für die SE einführen ${ }^{23}$. Die Anwendbarkeit der Quotenvorgabe auf die SE betrifft immerhin eine Reihe prominenter börsennotierter und paritätisch mitbestimmter Großunternehmen ${ }^{24}$.

Das Gesetz geht dagegen davon aus, die Quote könne gemäß $\ 17$ Abs. 2 SEAG-Entwurf und $\$ 24$ Abs. 3 SEAG-Entwurf auch auf die dualistische und monistische SE erstreckt werden. Die vom europäischen Gesetzgeber für die Mitbestimmung in der SE vorgesehene Verhandlungslösung enthalte einen weiten Gestaltungsspielraum, der zur Implementierung der Quote genutzt werden könne ${ }^{25}$. Letztere Auffassung ist überzeugend.

21 BT-Drucks. 18/3784, S. $141 \mathrm{f}$ zu $\ 17$ Abs. 2 SEAG-Entwurf: „Nach Art. 40 Abs. 2 SEVO werden alle Mitglieder des Aufsichtsorgans von der Hauptversammlung bestellt. Dabei ist das Ergebnis der aufgrund der Vereinbarung über die Mitbestimmung der Arbeitnehmer erfolgten Zusammensetzung der Arbeitsnehmerseite des Aufsichtsorgans zu berücksichtigen. Die Bestellung kann nur rechtswirksam erfolgen, wenn die Voraussetzungen des Absatzes 2 Satz 1 erfüllt sind." (Hervorhebung durch Verf.).

22 PÜtz/Weckes, Geschlechterquote, Report Nr. 1-2014 der Hans Böckler-Stiftung, 2014, S. 11; kritisch auch Kraft/Redenius-Hövermann, AG 2012, 28, $31 \mathrm{f}$.

23 Pütz/Weckes, Geschlechterquote, Report Nr. 1-2014 der Hans Böckler-Stiftung, 2014, S. 11.

24 Allianz SE, BASF SE, Bilfinger SE, E.ON SE, MAN SE, Porsche Holding SE, SGL Carbon SE, vgl. PüTz/WEckes, Geschlechterquote, Report Nr. 1-2014 der Hans Böckler-Stiftung, 2014, S. 5.

25 BT-Drucks. 18/3784, S. 141: „Der europäische Gesetzgeber hat die Rahmenbedingungen für die Verhandlungen festgelegt und den Verhandlungsparteien im Übrigen einen weiten Gestaltungsspielraum gelassen. [...] Diesen Gestaltungsspielraum gilt es auch zu nutzen, um gemeinsam mit der Anteilseignerseite eine der Quotenregelung entsprechende geschlechtergerechte Besetzung des paritätisch mitbestimmten Aufsichts- oder Verwaltungsorgans der börsennotierten SE nach Absatz 2 Satz 1 zu erreichen.“; zur 
2. Quote als Bestandteil des sekundären Gesellschaftsstatuts (Art. 9 Abs. 1 lit.c SE-VO)

Die Regelungskompetenz des deutschen Gesetzgebers zur Einführung der Quote im Aufsichts- oder Verwaltungsorgan einer SE mit Satzungssitz im Inland ergibt sich aus der Kollisionsnorm des Art. 9 Abs. 1 lit.c i) und ii) SE-VO. Diese Bestimmung regelt das auf die SE anzuwendende Recht im vertikalen Regelungskonflikt zwischen der (vorrangigen) supranationalen SEVO und dem (nachrangigen) mitgliedstaatlichen Gesellschaftsrecht. Das primäre Personalstatut einer SE ist hiernach die SE-VO selbst, das sekundäre Personalstatut für eine SE mit Satzungssitz im Inland setzt sich aus dem deutschen SE-Ausführungsgesetz (SEAG) sowie dem deutschen Aktienrecht (AktG 1965 plus diesbezügliche Rechtsprechungsregeln) zusammen ${ }^{26}$.

Das primäre Gesellschaftsstatut enthält keine Quotenregelung, und zwar weder für das Aufsichtsorgan in der dualistischen SE (vgl. Art. 40 SE-VO) noch für das Verwaltungsorgan in der monistischen SE (vgl. Art. $43 \mathrm{SE}-\mathrm{VO}$ ). Damit ist der Weg frei für eine Quotenregelung auf Ebene des sekundären Gesellschaftsstatuts. Denn für die „nicht durch diese Verordnung geregelten Bereiche oder, sofern ein Bereich nur teilweise geregelt ist, in Bezug auf die nicht von dieser Verordnung erfassten Aspekte“, eröffnet Art. 9 Abs. 1 lit. c SE-VO den Mitgliedstaaten eine entsprechende Regelungsautonomie ${ }^{27}$. Diese ist Ausdruck einer „politisch gewollten Nationalisierung der SE“"28. „Im Sinne einer Faustformel“ gilt daher mit Hommelhoff/Teichmann, „dass bei der heutigen nur noch rudimentären Regelung der meisten Rechtsbereiche nur selten von einer abschließenden Regelung [der SE-VO] ausgegangen werden kann.“"

\section{Keine Sperrwirkung der SE-Mitbestimmungsrichtlinie (RL 2001/86/EG)}

Die SE-Mitbestimmungsrichtlinie (RL 2001/86/EG, im Folgenden SE-RL) entfaltet ebenfalls keine Sperrwirkung in Bezug auf eine Quotenreglung in den $\int \mathbb{1 7}$, 24 SEAG-Entwurf. Sie ist zwar lex specialis für die Beteiligung der

Quotenregelung in der Europäischen Aktiengesellschaft vgl. Teichmann/Rüв, Die gesetzliche Geschlechterquote in der Privatwirtschaft, BB 2015, 259, $263 \mathrm{ff}$ (im Erscheinen).

26 Zum Zusammenspiel von primärem und sekundärem Personalstatut einer Societas Europaea CASPER/Weller, NZG 2009, $681 \mathrm{ff}$.

27 Es handelt sich um eine „dynamische Verweisung“ auf das jeweils geltende mitgliedstaatliche Aktienrecht, Hommelhoff/Teichmann, in: Lutter/Hommelhoff, SE-Kommentar, 2008, Art. 9 Rdn. 55.

28 Casper, FS Ulmer, 2003, S.51, 58; zustimmend Hommelhoff/Teichmann, aaO (Fn. 27), Art. 9 SE Rdn. 50.

29 Hommelhoff/Teichmann, aaO (Fn. 27), Art. 9 SE Rdn. 47. 
Arbeitnehmer in der SE; dabei geht es ausweislich der Legaldefinition in Art. 2 lit. h SE-RL um das „Verfahren (...), durch das die Vertreter der Arbeitnehmer auf die Beschlussfassung innerhalb der Gesellschaft Einfluss nehmen können." Art. 4 SE-RL regelt in seinem Absatz 2 enumerativ Inhalte der Mitbestimmungsvereinbarung; darin können insbesondere die Zusammensetzung, die Befugnisse und die Verfahrensordnung des Aufsichts- oder Verwaltungsorgans, in dem die Arbeitnehmer vertreten sind, geregelt werden.

Allerdings stehen die in Art. 4 Abs. 2 SE-RL genannten Inhalte der Mitbestimmungsvereinbarung erstens unter dem Vorbehalt des Rechts des Mitgliedstaats, in dem die SE ihren Satzungssitz haben wird (Art. 6 SE-RL) sowie zweitens unter dem Vorbehalt der „Autonomie der Parteien“ (Art. 4 Abs. 2 Satz 1 SE-RL: ,unbeschadet der Autonomie der Parteien“). Der Privatautonomie wird also Vorrang vor dem Inhaltskatalog des Art. 4 Abs. 2 SE-RL eingeräumt; dies erhellt, dass es sich bei dem Katalog um eine nicht abschließende Regelung handelt, welche die Regelung einer Quote im sekundären Personalstatut der SE nicht ausschließt.

Eine weitere Überlegung bestätigt diesen Befund: In der SE-Mitbestimmungsrichtlinie hätte eine Quote von der EU kompetenziell nicht statuiert werden können: Die SE-RL wurde nämlich auf die Abrundungskompetenz des Art. 308 EGV (= Art. 352 AEUV) gestützt ${ }^{30}$. Eine Quotenregelung auf Ebene des EU-Sekundärrechts müsste jedoch, wenn man eine solche erlassen wollte, auf den spezielleren Art. 157 Abs. 3 AEUV gestützt werden ${ }^{31}$. Die SE-Mitbestimmungs-RL kann mithin keine Sperrwirkung in Bezug auf eine Quotenregelung entfalten, weil ihr Anwendungsbereich sich darauf nicht erstreckt.

Bestätigt wird die Regelungskompetenz des nationalen Gesetzgebers in Bezug auf die Quotenreglung schließlich durch Art. 9 Abs. 3 SE-VO, wonach die SE in Bezug auf ihre Geschäftstätigkeit den besonderen Vorschriften des einzelstaatlichen Rechts unterliegt. Auch wenn hier primär Regulierungsvorgaben im Bank- und Versicherungsrecht gemeint sind, so ist Art. 9 Abs. 3 SE-VO doch Ausdruck des Prinzips, dass eine supranationale Rechtsform keine Sonderbehandlung oder Privilegierung gegenüber nationalen Rechtsformen beansprucht ${ }^{32}$.

Bilanz: Der deutsche Gesetzgeber hat die Kompetenz, eine Geschlechterquote für den Aufsichts- bzw. Verwaltungsrat einer SE zu statuieren.

30 Erwägungsgrund 17 SE-RL.

31 So denn auch der Vorschlag der EU-Kommission für eine Geschlechterquoten-RL für Aufsichtsräte börsennotierter Gesellschaften, COM(2012) 614/5, S. 11; hierzu Jung, BB 2013, 387, 388.

32 Hommelhoff/Teichmann, aaO (Fn. 27), Art. 9 SE Rdn. 60. 


\section{Die Anwendbarkeit auf Auslandsgesellschaften- der kollisionsrechtliche Rabmen}

Neben der SE erweist sich die Anwendbarkeit der Quotenregelung auf Auslandsgesellschaften mit Tätigkeitsschwerpunkt im Inland als problematisch. $\mathrm{Zu}$ denken ist etwa an die Air Berlin PLC ${ }^{33}$, eine Gesellschaft englischen Rechts, die ihren Verwaltungssitz ebenso wie ihre hauptsächlichen wirtschaftlichen Aktivitäten in Deutschland hat. Die aus der Niederlassungsfreiheit (Art. 49, 54 AEUV) abgeleitete europäische Rechtsformwahlfreiheit gestattet freilich den Einsatz solcher EU-ausländischer Gesellschaften im Inland ${ }^{34}$. Paradigmatisch hierfür steht die Entscheidungskaskade Centros ${ }^{35}$, Überseering $^{36}$ und Inspire Art ${ }^{37}$. Allerdings beschränkt sich die Rechtsformwahlfreiheit auf das Gesellschaftsstatut. Davon nicht umfasst ist zum einen das Insolvenzstatut ${ }^{38}$. Dieses richtet sich anders als das Gesellschaftsstatut nicht nach dem Ort der Gründung (Gründungstheorie) sondern gemäß Art. 4 EuInsVO nach dem Mittelpunkt der hauptsächlichen Interessen des Schuldners, dem sog. Center of Main Interest (COMI), der bei Gesellschaften mit deren Verwaltungssitz zusammenfällt ${ }^{39}$. Gegen das ausländische Gesellschaftsstatut setzen sich darüber hinaus marktbezogene Inlandsregelungen durch, die dem Wettbewerb auf inländischem Territorium einen einheitlichen Rahmen geben, mithin das Kartell- und Lauterkeitsrecht ${ }^{40}$. Die reguläre Verweisung der Gründungstheorie auf das (ausländische) Gesellschaftsstatut wird schließlich durchbrochen über die Sonderanknüpfung von Eingriffsnormen der lex fori, zu denen auch die starre Aufsichtsratsquote zu rechnen ist.

33 Vgl. die Articles of Association of Air Berlin PLC, as adopted by special resolution on 7 June 2012 and as amended by special resolution on 18 June 2014, abrufbar unter www.airberlin.com (letzter Abruf: 15. 2. 2015).

34 Weller/Thomale, Companies, in: Basedow et al., Encyclopedia of European Private International Law, 2015.

35 EuGH, Urt. v. 9.3.1999, Rs. C-212/97, Slg. 1999, I-1459-Centros.

36 EuGH, Urt. v. 5.11.2002, Rs. C-208/00, IPRax 2003, 65 - Überseering.

37 EuGH, Urt. v. 30. 9.2003, Rs. C-167/01, NJW 2003, 3331 - Inspire Art.

38 Vgl. zur internationalen Zuständigkeit für Klagen des Insolvenzverwalters aus $\$ 64$ GmbHG gegen den Geschäftsführer analog Art. 3 EuInsVO jüngst EuGH, Urt. v. 4. 12. 2014, C-295/13 - „H“, Rdn. 17-24, sowie zur insolvenzrechtlichen Qualifikation des $\$ 64$ GmbHG im Rahmen des Art. 4 EuInsVO BGH, Vorlagebeschluss v. 2. 12. 2014, II ZR 119/14. Demnächst näher zur Abgrenzung von Gesellschafts- und Insolvenzstatut Thomale/Weller, Gläubigerschutz via Insolvenzstatut, 2015 (in Vorbereitung).

39 EuGH, Urt. v. 20. 10. 2011, Rs. C-396/09, Slg. 2011, I-09915 - Interedil.

40 Vgl. die Marktortanknüpfung in Art. 6 Rom II-VO. 


\section{Die Quotenregelung als Eingriffsnorm}

Im Folgenden soll dargelegt werden, dass die Quotenregelungen in $\$ 96$ Abs. 2 AktG-Entwurf und $\mathbb{S} \$ 17$ Abs. 2, 24 Abs. 3 SEAG-Entwurf eingriffsrechtlichen Charakter haben.

\section{Begriff und Wirkungsweise von Eingriffsnormen}

Im Internationalen Privatrecht wird zwischen allseitig-neutralen Kollisionsnormen und einseitig-zwingenden Eingriffsnormen unterschieden ${ }^{41}$. Letztere sind aufgrund ihres internationalen Geltungswillens unabhängig von der jeweiligen Verweisungsentscheidung, wie sie etwa von der Gründungstheorie in Bezug auf die Gründungsrechtsordnung einer Gesellschaft ausgesprochen wird, im Wege der Sonderanknüpfung anzuwenden ${ }^{42}$.

Ob eine Norm tatbestandlich einen eingriffsrechtlichen Charakter aufweist, ergibt sich durch Auslegung aus Perspektive der lex fori ${ }^{43}$. Eingriffsnormen sind nur solche Bestimmungen, die überindividuelle, öffentliche Interessen regeln (Gemeinwohlorientierung); sie müssen mithin über den bloßen Ausgleich widerstreitender Parteiinteressen hinausgehen ${ }^{44}$. Es handelt sich üblicherweise um Normen wirtschafts-, gesellschafts- und/oder sozialpolitischer Natur, die das Wirtschafts- oder Arbeitsleben beeinflussen ${ }^{45}$. Sie befinden sich regelmäßig auf der Schnittstelle zwischen Internationalem Privatrecht und Internationalem Öffentlichen $\mathrm{Recht}^{46}$. Eine positiv-rechtliche Definition von Eingriffsnormen findet sich im Internationalen Schuldvertragsrecht in Art. 9 Abs. 1 Rom I-VO; sie hat pars pro toto für das gesamte IPR Leitbildcharakter ${ }^{47}$ : „Eine Eingriffsnorm ist eine zwingende Vorschrift, deren Einhal-

41 von Hein, Münchener Komm. z. BGB, 6. Aufl., 2015, Einl. IPR Rdn. 286.

42 Schurig, Zwingendes Recht, „Eingriffsnormen“ und neues IPR, RabelsZ 54 (1990), $217(220)$.

43 Schurig, RabelsZ 54 (1990), 217, 236 f; Staudinger, in: Schulze u. a., BGB Handkomm., 8. Aufl., 2014, Art. 9 Rom I-VO Rdn. 4.

44 Wengler, Anknüpfung des zwingenden Schuldrechts im internationalen Privatrecht, ZVglRWiss 54, 168, 197; von Bar/Mankowski, Internationales Privatrecht I, 2. Aufl., 2003, \4 Rdn. 106; Schurig, Zwingendes Recht, „Eingriffsnormen“ und neues IPR, RabelsZ 54 (1990), 217, 227; Sonnenberger, Eingriffsrecht - das trojanische Pferd im IPR, IPRax 2003, 104, 107 ff; Weller, aaO (Fn. 9), Einl. GmbHG Rdn. 467.

45 Martiny, Münchener Komm. z. BGB, 6. Aufl., 2015, Rom I-VO, Art. 9 Rdn. 11; vgl. zu Art. 34 EGBGB aF BT-Drucks. 10/504, 83.

46 Martiny, aaO (Fn. 45), Rom I-VO, Art. 9 Rdn. 1.

47 Art. 9 Rom I-VO sei zusammen mit Art. 16 Rom I-VO „Ausdruck eines allgemeinen kollisionsrechtlichen Prinzips“, weshalb die Definition des Art. 9 Rom I-VO verallgemeinerungsfähig ist, MaGnus, in: Staudinger, BGB, Neubearb. 2011, Art. 9 Rom I-VO Rdn. 15. 
tung von einem Staat als so entscheidend für die Wabrung seines öffentlichen Interesses, insbesondere seiner politischen, sozialen oder wirtschaftlichen Organisation, angesehen wird, dass sie ungeachtet des nach Maßgabe dieser Verordnung auf den Vertrag anzwwendenden Rechts auf alle Sachverbalte anzuwenden ist, die in ibren Anwendungsbereich fallen."

$\mathrm{Ob}$ und wie Eingriffsnormen neben dem anwendbaren Recht, also der lex causae, Beachtung finden, wird danach unterschieden, ob es sich um Normen der lex fori oder forumsfremde Normen eines Drittstaates handelt ${ }^{48}$. Während die Heranziehung forumsfremder Normen umstritten ist ${ }^{49}$, sind forumseigene Eingriffsnormen - und um eine solche würde es sich bei $\$ 96$ Abs. 2 Satz 1 AktG-Entwurf handeln - nach allgemeiner Ansicht im Wege einer Sonderanknüpfung anwendbar ${ }^{50}$. Diese setzt freilich voraus, dass in sachlicher Hinsicht ein hinreichender Konnex des Regelungszwecks der Eingriffsnorm zum Sachverhalt besteht und in räumlicher Hinsicht ein ausgeprägter Inlandsbezug gegeben ist $\mathrm{t}^{51}$.

\section{Beispiele für Eingriffsnormen im Unternehmensrecht}

Eingriffsnormen sind im Unternehmensrecht keine Seltenheit. Insbesondere das Außenwirtschaftsgesetz (AWG) setzt sich auch gegen ein ausländisches Gesellschaftsstatut beim Erwerb von Gesellschaftsanteilen durch ${ }^{52}$. So ordnet $\$ 15$ Abs. 3 i. V.m. $\$ 4 A^{A W G}{ }^{53}$ an, dass ein dingliches Vollzugsgeschäft, das einen Beteiligungserwerb an einem inländischen Unternehmen in einem si-

48 Wengler, Anknüpfung des zwingenden Schuldrechts im internationalen Privatrecht, ZVglRWiss 54, 168, 171.

49 Vgl. Art. 9 Abs. 3 Rom I-VO einerseits sowie BGHZ 59, 82 (Nigerianische Masken) andererseits; ferner Sonnenberger, Münchener Komm. z. BGB, 5. Aufl., 2010, Rdn. 39; von Hein, aaO (Fn. 41), Einl. IPR Rdn. 291.

50 Vgl. für das Vertragsrecht Art. 9 Abs. 2 Rom I-VO; allgemein Martiny, aaO (Fn. 45), Rom I-VO, Art. 9 Rdn. 27; Schurig, RabelsZ 54 (1990), 217, 223: „Dass ein Staat eigene Bestimmungen, denen er eine besondere Anknüpfungsnorm zuordnet, dann, wenn die Anknüpfung erfüllt ist, auch anwendet, ist eine bare Selbstverständlichkeit und immer schon als solche angesehen worden (auch von Savigny).“

51 Schurig, Zwingendes Recht, „Eingriffsnormen“ und neues IPR, RabelsZ 54 (1990), 217, 236.

52 Remien, Außenwirtschaftsrecht in kollisionsrechtlicher Sicht, RabelsZ 54 (1990), 431, 464; Martiny, Europäisches Internationales Vertragsrecht, ZEuP 2006, 60, 87; allerdings unterliegen Außenwirtschaftsregulierungen „der ausschließlichen Kompetenz der Gemeinschaft (Artt. 205 AEUV), so dass Ein- und Ausfuhrbeschränkungen nach dem AWG oder der AußenwirtschaftsVO - auch wenn sie als Eingriffsnormen zu qualifizieren sind - dahinter zurücktreten“, DoEHNER, in: Hüßtege/Mansel, BGB RomVerordnungen, 1. Aufl., 2014, Art. 9 Rom I-VO Rdn. 27.

$53 \rrbracket 15$ Abs. 3 AWG entspricht $\$ 32$ Abs. 2 AWG a. F., vgl. Hensel/Pohl, AG 2013, 849, 859. 
cherheitsrelevanten oder strategischen Wirtschaftssektor zum Gegenstand hat (z. B. Unternehmen der Daseinsvorsorge), der Meldepflicht und einem Verbotsvorbehalt unterfällt und während der behördlichen Prüfungsphase schwebend unwirksam ist ${ }^{54}$. Da die Normen des AWG auf wirtschaftspolitische Steuerung abzielen, haben sie grundsätzlich internationalen Geltungswillen ${ }^{55}$. Daher findet $\$ 15$ Abs. 3 AWG auch dann Anwendung, wenn das inländische Unternehmen, an dem die Beteiligung erworben werden soll, nach einem ausländischen Gesellschaftsstatut organisiert ist ${ }^{56}$.

Erhebliche Einwirkungen auf das Gesellschaftsstatut hat zudem das Luftverkehrsnachweissicherungsgesetz (LuftNaSiG). Dieses will eine 40\%-Mindestbeteiligung von EU-inländischen Aktionären an einem Luftfahrtunternehmen sicherstellen und macht insofern Vorgaben für die Ausgestaltung der Aktien ( $\mathbb{\$} 2 \mathrm{ff}$ LuftNaSiG) und für Kapitalmaßnahmen der Gesellschaft ( $\$ 4$ Luft$\mathrm{NaSiG})$. Obwohl $\$ 1$ LuftNaSiG seinem Wortlaut nach lediglich Aktiengesellschaften mit Sitz im Inland adressiert, ist es als Eingriffsrecht zu qualifizieren und dementsprechend auch auf ausländische Aktiengesellschaften anzuwenden, die im Inland eine Luftverkehrsbetriebsgenehmigung erhalten möchten. Daher werden seine Vorgaben auch von der dem englischen Gesellschaftsstatut unterliegenden Air Berlin PLC in entsprechender Anwendung beachtet $^{57}$.

Ein weiteres (allerdings umstrittenes) Beispiel für Eingriffsnormen sind die Vorschriften zur unternehmerischen Mitbestimmung, welche die Beteiligung der Arbeitnehmer im Aufsichtsrat regeln, etwa das MitbestG 1976 ${ }^{58}$. Die un-

54 Vgl. Weller, ZIP 2008, 857, 861.

55 Staudinger, in: Ferrari/Kieninger/Mankowski u.a., Internationales Vertragsrecht, 2. Aufl., 2011, Art. 9 Rom I-VO, Rdn. 25.

56 Vgl. allgemein zu Sonderanknüpfung von Verboten mit Nichtigkeitsfolge im AWG Martiny, aaO (Fn. 45), Art. 9 Rom I-VO Rdn. 65.

$57 \mathrm{Vgl}$. unter http://ir.airberlin.com (zuletzt abgerufen am 16.2.2015): „Airberlin ist eine Aktiengesellschaft britischen Rechts (public limited company) und unterliegt daher nicht dem LuftNaSiG. Um gleichwohl die Einhaltung der luftverkehrsrechtlichen Eigentums- und Kontrollvorschriften sicherstellen zu können, sieht die Satzung von airberlin zunächst vor, dass Aktionäre der airberlin nur dann in das Aktienregister (sog. „CI Register") eingetragen werden, wenn sie der Gesellschaft ihre Nationalität offen legen. Sollten Aufrechterhaltung oder Erteilung von Betriebsgenehmigung oder einzelnen Verkehrsrechten gefährdet sein, können die Directors von airberlin verschiedene Maßnahmen ergreifen. Hierzu gehören neben der Verweigerung der Zustimmung zur Übertragung von Aktien und zur Eintragung von Aktionären etwa Auskunftsverlangen gegenüber Aktionären, die Beschränkung der Ausübung von Stimmrechten oder auch die Aufforderung zum Verkauf von Aktien. Schließlich können die Directors - im Falle des fruchtlosen Verstreichens einer Frist zum Verkauf - die Aktien selbst im Namen des Aktionärs verkaufen.“

58 Vgl. Grossfeld, in: Erlinghagen, Internationales Unternehmensrecht und Mitbestimmung, JZ 1993, 217 ff; Junker, Das Internationale Arbeitsrecht im Spiegel der Recht- 
ternehmerische Mitbestimmung unterliegt zwar dem Gesellschaftsstatut ${ }^{59}$. Sofern verweisungsrechtlich ausländisches Recht anwendbar ist, kommen die $\mathbb{S} \mathbb{S} 1 \mathrm{ff}$ MitbestG deshalb über das Internationale Gesellschaftsrecht nicht zur Anwendung ${ }^{60}$. Allerdings verwirklichen die Mitbestimmungsnormen (auch) sozialpolitische Ordnungsvorstellungen, was ihnen internationalen Geltungswillen und damit den Charakter von Eingriffsrecht verleiht, welches das Internationale Gesellschaftsrecht überlagert ${ }^{61}$. Daher können die Regeln über die unternehmerische Mitbestimmung im Wege der Sonderanknüpfung ungeachtet des ausländischen Gesellschaftsstatuts angewendet werden, sofern eine Auslandsgesellschaft ihren effektiven Verwaltungssitz im Inland hat und somit eine hinreichende Inlandsbeziehung besteht $t^{62}$.

Gegen eine solche Sonderanknüpfung wird zwar eingewandt, $\mathbb{S} 1$ MitbestG 1976 erfasse lediglich bestimmte inländische Gesellschaftsformen (AG, $\mathrm{KGaA}, \mathrm{GmbH}, \mathrm{eG})^{63}$. Zudem sprächen rechtstechnische Anwendungsschwierigkeiten gegen die eingriffsrechtliche Erstreckung der unternehmerischen Mitbestimmung auf ausländische Gesellschaften ${ }^{64}$.

sprechung, 50 Jahre Bundesarbeitsgericht, 1197, 1214 f; Kindler, Münchener Komm. z. BGB, 5. Aufl., 2010, Internationales Gesellschaftsrecht Rdn. 597.

59 Zimmer, Internationales Gesellschaftsrecht, 1996, S. 143 f.

60 МеRкт, Unternehmensmitbestimmung für ausländische Gesellschaften?, ZIP 2011, $1237,1239$.

61 Franzen, Niederlassungsfreiheit, internationales Gesellschaftsrecht und Unternehmensmitbestimmung, RdA 2004, 257, 259; GrossFELD, in: Staudinger, Internationales Gesellschaftsrecht, Neubearb. 1998, Rdn. 460; Kindler, ZHR 2015, Heft 2/3; Weller, FS Hommelhoff, S.1275, 1289 f; differenzierend Zimmer, aaO (Fn. 59), S.148 ff; aA SANDROCK, Gehören die deutschen Regelungen über die Mitbestimmung auf Unternehmensebene wirklich zum deutschen ordre public?, AG 2004, 57, 64: Aufgrund der vielen Mängel des Mitbestimmungsgesetzes könnten jene Regelungen nicht „als staatstragend“ bezeichnet werden; vgl. zudem WindbichleR/Bachmann, Corporate Governance und Mitbestimmung als „wirtschaftlicher ordre public“, S.797, 804: „Die Begründung dafür, die Mitbestimmung sei von allgemeiner gesellschaftspolitischer Bedeutung und diene dem Wohle der Allgemeinheit, stützt sich unmittelbar auf die Mitbestimmungsentscheidung des BVerfG, vernachlässigt aber einen dort zu findenden Einschub: Es heißt „- ungeachtet ihrer Ausgestaltung im einzelnen -“.

62 Franzen, Niederlassungsfreiheit, internationales Gesellschaftsrecht und Unternehmensmitbestimmung, RdA 2004, 257, 259; so auch schon Birk, Mitbestimmung und Kollisionsrecht, RIW 1975, 589, 594 f; WeLler, FS Hommelhoff, Unternehmensmitbestimmung für Auslandsgesellschaften, S. 1275, $1288 \mathrm{ff}$; zustimmend KINDLER, ZHR 2015, Heft. 2/3.

63 WeLLER, Unternehmensmitbestimmung für Auslandsgesellschaften, FS Hommelhoff, S. 1275, 1276; Zimmer, aaO (Fn. 59), S. $158 \mathrm{ff}$ sieht hierin eine Grenze der kollisionsrechtlichen Sonderanknüpfung, die auch nicht im Wege einer Analogie auf Gesellschaften mit effektivem Verwaltungssitz im Inland überwunden werden kann.

64 Zimmer, Nach „Inspire Art“: Grenzenlose Gestaltungsfreiheit für Unternehmen?, NJW 2003, 3585, 3591; HoRn, Deutsches und europäisches Gesellschaftsrecht und die EuGH-Rechtsprechung zur Niederlassungsfreiheit - Inspire Art, NJW 2004, 893, 
Diese Gegenargumente überzeugen jedoch aus kollisionsrechtlicher Sicht nicht: Der entgegenstehende Wortlaut und eine etwaige fehlende planwidrige Regelungslücke zur analogen Anwendung des $\$ 1$ MitbestG 1976 auf ausländische Gesellschaften mit Verwaltungssitz im Inland sind deshalb unbeachtlich, weil die durch $₫ 1$ MitbestG 1976 in Bezug genommenen inländischen Gesellschaften von bestimmten ausländischen Gesellschaftsformen gemäß Art. 3 EGBGB im Wege der anerkannten allgemeinen IPR-Figur der Substitution ersetzt werden können ${ }^{65}$. Anders als eine Analogie erfordert die Substitution nämlich keine planwidrige Regelungslücke ${ }^{66}$, sondern lediglich die bei $\$ 1$ MitbestG gegebene - Offenheit der Sachnorm ${ }^{67}$, innerhalb derer ein bestimmtes normativ geprägtes Tatbestandsmerkmal substituiert wird, sofern die ausländische und die zu substituierende inländische Rechtsfigur funktional vergleichbar sind ${ }^{68}$.

\section{Der eingriffsrechtliche Charakter der $\iint 96$ Abs. 2 AktG-Entwurf und 24 Abs. 3 SEAG-Entwurf}

Ob die $\int \mathbb{S} 96$ Abs. 2 AktG-Entwurf und 24 Abs. 3 SEAG-Entwurf einen international zwingenden Charakter aufweisen, ist ausschließlich abstrakt „vom Gesetz her" zu bestimmen ${ }^{69}$. Darin liegt eine fundamentale Abweichung vom klassischen Internationalen Privatrecht, das grundsätzlich von dem konkreten Sachverhalt ausgehend versucht, die passenden Sachrechtsnormen am Sitz des Rechtsverhältnisses aufzufinden ${ }^{70}$. Solche „Gesetze von streng positiver, zwingender Natur, die eben wegen dieser Natur zur freien Behandlung, unabhängig von den Grenzen verschiedener Staaten, nicht geeignet sind“, können allein durch Auslegung ermittelt werden, bei der die zu Tage getretene „Absicht des Gesetzgebers“ eine entscheidende Rolle spielt ${ }^{71}$.

900; Junker, Sechsundsiebzig verweht - Die deutsche Mitbestimmung endet in Europa, NJW 2004, 728, 729; MERKT, Unternehmensmitbestimmung für ausländische Gesellschaften?, ZIP 2011, 1237, 1239, 1241 f; aA zur möglichen rechtstechnischen Umsetzung Franzen, Niederlassungsfreiheit, internationales Gesellschaftsrecht und Unternehmensmitbestimmung, RdA 2004, 257, 261.

65 Franzen, RdA 2004, 257, 260; Kindler, ZHR 2015, Heft 2/3.

66 Diese Voraussetzungen einer Analogie lägen für $\mathbb{\$} 1$ MitbestG 1976 hinsichtlich der Anwendung auf ausländische Gesellschaften nicht vor vgl. МеRкт, Unternehmensmitbestimmung für ausländische Gesellschaften?, ZIP 2011, 1237, 1239; Zimmer, aaO (Fn. 59), S. $161 \mathrm{ff}$.

67 Näher Weller, FS Hommelhoff, S. $1275 \mathrm{ff}$.

68 Zur Substitution vgl. von Bar/Mankowski, aaO (Fn. 44), $\$ 7$ Rdn. 239 ff.

69 Formulierung nach Kropholler, IPR, 6. Aufl., 2006, 21.

70 Zur klassischen IPR-Methode Weller, IPRax 2011, 429, 431.

71 Savigny, System, Band VIII, 1849, 33 f; hierzu W.-H. Roth, FS Kühne, 2009, S. 859 ff. 
Ein ausdrücklicher Gesetzgeberwille lässt sich der Gesetzesbegründung zur Frage der allgemeinen internationalen Anwendbarkeit der Quotenregelung nicht entnehmen. Zwar geht die summarische Wirkungsanalyse der Begründung davon aus, von der Regelung seien „naturgemäß die großen Publikumsgesellschaften in der Rechtsform der Aktiengesellschaften (AG) und Kommanditgesellschaften auf Aktien (KGaA) betroffen (ca. 108 an der Zahl)."72 Dieser lediglich beispielhaften Benennung des unmittelbaren nationalen Anwendungsfeldes kann jedoch kein impliziter Ausschluss eines international zwingenden Anwendungsbereichs entnommen werden. Deshalb sind - wie dies regelmäßig bei der Bestimmung eines Eingriffsnormcharakters der Fall ist - Sinn und Zweck der Quotenregelung zu würdigen.

\section{a) National und international zwingender Charakter der Quotenregelung}

Eine notwendige Bedingung für international zwingende Eingriffsnormen ist ihr indispositiver Charakter. Denn Regeln, die bereits unter privatautonomem Änderungsvorbehalt stehen, können sinnfälligerweise erst recht keine parteiautonome Abrede oder zwingendes Kollisionsrecht überspielen. $\$ \mathbb{S} 96$ Abs. 2 AktG-Entwurf und 24 Abs. 3 SEAG-Entwurf erfüllen diese Voraussetzung, weil sie nicht zur Disposition der betroffenen Gesellschaft stehen.

Der indispositive Charakter einer Norm allein reicht jedoch nicht hin, um sie auch als international zwingende Eingriffsnorm zu qualifizieren. Dazu muss die Norm vielmehr zusätzlich einen öffentlichen, außenprivatrechtspolitischen $^{73}$ Zweck verfolgen, der sie ihres allgemeinen privatrechtlichen Wirkungszusammenhangs enthebt, indem sie „nicht allein um der Personen Willen, welche die Träger der Rechte sind“ gilt ${ }^{74}$.

\section{b) Lediglich Förderung privater Interessen?}

In dieser Hinsicht könnten die $\$ \int 96$ Abs. 2 AktG-Entwurf und 24 Abs. 3 SEAG-Entwurf prima facie freilich als international nachgebend zu qualifizieren sein. Denn die Gesetzesbegründung gibt als zentrales Motiv für die neue Quotenregelung an, „dass die Wettbewerbsfähigkeit der Unternehmen gesteigert werden kann, wenn der Anteil von Frauen an Führungspositionen zunimmt. “75 Damit wird ein Argumentationstopos aufgegriffen, der die Quotendebatte schon länger beeinflusst: Unter der Prämisse, dass die Eignung für

73 Begriff nach Wengler, BGB-RGRK, 12. Aufl., 1981, Band VI/1, 269.

74 SAvigny, aaO (Fn. 71), 36. Vgl. aus neuerer Zeit BAG, BAGE 63, 17, 32.

75 BT-Drucks. 18/3784, S. 45. 
und das Interesse an Aufsichtsratspositionen unter Männern und Frauen gleichverteilt $\operatorname{sind}^{76}$, zeugt die abseits jeder plausiblen Standardabweichung liegende Unterrepräsentierung von Frauen in Aufsichtsräten davon, dass diese Positionen derzeit nicht nach leistungsbezogenen Kriterien vergeben werden $^{77}$. Quoten stellen folglich ein Mittel dar, um leistungsfeindliche Auswahlprozesse effizienter zu gestalten, indem sie das Potential der aus sachfremden Gründen verdrängten Bewerberinnenkohorte heben. Die vorgeschlagene Quotenregelung bezweckt unter diesem Vorzeichen lediglich die Optimierung der deutschen Aufsichtsräte ${ }^{78}$ : Den Aktionären einer deutschen Aktiengesellschaft wird gleichsam der Vorteil einer effizienten Kontrollverwaltung aufgedrängt. Sie werden insoweit ähnlich wie Verbraucher, Arbeitnehmer und andere Schutzbedürftige in ihrem wohlverstandenen Eigeninteresse vor ihrer eigenen Irrationalität bewahrt ${ }^{79}$. Einer solchen, nur aus verhaltensökonomischen Gründen indispositiv ausgestalteten, Vorschrift ließe sich kaum ein international zwingender Geltungswille entnehmen. Denn die Quotenregelung diente in diesem Fall allein oder zumindest vorrangig privaten Interessen.

\section{c) Sozialpolitische und marktordnende Stoßrichtung der Quotenregelung}

Eine genauere Analyse ergibt jedoch, dass die vorgeschlagene Quotenregelung den beschriebenen Effizienzaspekt in einen größeren sozialpolitischen Wirkungszusammenhang einbettet. So betont das Gesetz zunächst, dass es ihm mit der geplanten Effizienzsteigerung um den „Wirtschaftsstandort Deutschland" gehe, der von der "gesamtwirtschaftlich sinnvollen“ Quotenregelung profitieren werde ${ }^{80}$. Hier entfernt sich das Augenmerk bereits von den unmittelbar betroffenen Aktionären und Aktiengesellschaften und nimmt stattdessen eine marktordnende Perspektive ein: Entscheidend ist Deutschland als Ort der unternehmerischen Betätigung, nicht die Gesellschaftsverfassung nach deutschem Gesellschaftsstatut. Die Quotenregelung soll also ähnlich wie etwa

76 Siehe dazu Thomale, Gleichstellung und Besserstellung, JuS 2015, Editorial (im Erscheinen).

77 Vgl. zB Klesse/Berke/Kroker/Wildhagen, Aufsichtsräte Große Namen, aber kaum Experten, wiwo 8.12.2011; Groll, Was bringt die Frauenförderung?, zeitonline, 13.1.2015.

78 Konkret erhofft sich der Gesetzgeber von einer größeren Geschlechterdiversität in Aufsichtsräten eine „bessere Unternehmens- und Verwaltungsführung (corporate und public governance)“, die „Fehlentscheidung verhindern kann“, vgl. BT-Drucks. 18/378, S. 55 .

79 Für eine deutschsprachige Aufbereitung des in der amerikanischen Institutionenökonomie seit den 50er Jahren bekannten Tops der „bounded rationality“ siehe zuletzt: Schmolke, Grenzen der Selbstbindung im Privatrecht, 2014, $116 \mathrm{ff}$.

80 BT-Drucks. 18/3784, S. 45. 
AWG, KWG, UWG etc. einen Ordnungsrahmen für in Deutschland tätige Unternehmen bereitstellen ${ }^{81}$.

Diese ihrem Wesen nach öffentlich-rechtliche Stoßrichtung des Gesetzes tritt noch deutlicher hervor, wenn dieses feststellt, dass es „explizit gleichstellungspolitische Ziele verfolgt." 82 Genauer soll „den Benachteiligungen von Frauen entgegengewirkt werden, gleichzeitig geht es um die Ermutigung von Männern, Maßnahmen zur besseren Vereinbarkeit von Familie und Beruf für sich in Anspruch zu nehmen. " ${ }^{83}$ Davon ausgehend verspricht sich das Gesetz von der Quotenregelung einen Trickle-down-Effekt in dem Sinne, dass in Deutschland die „Unternehmenskultur hin zu mehr Arbeitnehmerfreundlichkeit und Flexibilität" ${ }^{44}$ verändert werden soll. Alle diese sozialpolitischen Zielsetzungen greifen erstens weit über den konkreten privatrechtlichen $\mathrm{Zu}$ sammenhang der Aufsichtsratswahl hinaus und sind zweitens orts- im Gegensatz zu (gesellschafts-)statutsbezogen, was entscheidend für eine Eingriffsnorm spricht.

Hinzu kommt drittens, dass das Gesetz "die nationale und internationale Wettbewerbsfähigkeit von Unternehmen "85 nachhaltig fördern möchte. Sähe man hierin lediglich die Wiederholung des Vertrauens des Gesetzes in die effizienzsteigernde Wirkung der Quotenregelung ${ }^{86}$, wäre dies redundant. Naheliegender erscheint vielmehr, dass sich das Gesetz an dieser Stelle implizit zum Vorwurf der Inländerdiskriminierung verhält. Die Quote soll kein Wettbewerbsnachteil der in jedem Fall betroffenen deutschen Unternehmen sein. Dies ist jedenfalls dann aus deutscher Sicht kein Problem, falls die Regelung ohnehin nur Vorteile bietet, worauf das Gesetz, wie gezeigt, auch vertraut. Gleichwohl handelt es sich bei der Quotenregelung immerhin formal um eine Beschränkung oder einschränkende Bestimmung des Aufsichtsratswahlrechts als Splitter des Aktieneigentums, die insbesondere i. V.m. den bestehenden Vorgaben der unternehmerischen Mitbestimmung auch als Wettbewerbsnachteil gesehen werden $\mathrm{kann}^{87}$. Um im Angesicht dieser Zweifel einer Inländerdiskriminierung prinzipiell entgegenzutreten, drängt sich ein internationa-

81 Mit derselben Begründung ist ebenso die Frage des Schutzes von Kleinaktionären gegen einen Widerruf der Börsenzulassung nicht an das Gesellschaftsstatut, sondern an den Anlageort und das dort gültige Börsen- und Kapitalmarktrecht zu knüpfen: Thomale, ZGR 2013, 686, $721 \mathrm{f}$. So nun auch: BGH, Beschl. v. 8. 11.2013, II ZB 26/12 - Frosta; hierzu AUER, JZ 2015, $71 \mathrm{ff}$.

82 BT-Drucks. 18/3784, S. 55.

83 BT-Drucks. 18/3784, S. 55.

84 BT-Drucks. 18/3784, S. 55.

85 BT-Drucks. 18/3784, S. 55. Hervorhebung von den VERF.

86 Siehe oben.

87 Die starre Mindestquote für die Besetzung von Aufsichtsräten wird teilweise sogar als verfassungswidrig eingestuft, vgl. HabersaCk/Kersten, BB 2014, 2819, 2830, Thesen 4 und 5. 
ler Anwendungsbefehl zusätzlich auf: Sobald die Quotenregelung räumlich auf den gesamten Wirtschaftsstandort Deutschland erstreckt wird, gelten für ausländische wie für deutsche Unternehmen dieselben Wettbewerbsbedingungen - gleichviel, ob und inwieweit die Quotenregelung den von ihr betroffenen Unternehmen Vor- oder Nachteile bietet. Dies aktiviert und fördert zugleich den unter Effizienzgesichtspunkten wünschenswerten Wettbewerb der Rechtsformen. Die unternehmerische Entscheidung, ob ein Großunternehmen mit einem Verwaltungssitz in Deutschland eher in Form einer PLC, einer SA oder einer AG zu organisieren ist ${ }^{88}$, sollte der gesellschaftspolitisch motivierten Geschlechtergleichstellung des Gesetzes indifferent gegenüberstehen. Genau dies hält das Gesetz mit Blick auf den vertikalen Wettbewerb der nationalen AG mit der supranationalen SE selbst in $\mathbb{S} 17$ Abs. 2 und 24 Abs. 3 SEAG-Entwurf fest. Dieser wettbewerbsorientierte Gleichstellungsgedanke, der im Gesetz bereits angelegt ist, wird mithin lediglich sinnfällig vollendet, wenn man ihn auch auf den horizontalen Wettbewerb der autonomen Rechtsformen untereinander erweitert ${ }^{89}$.

Insgesamt ist mithin festzuhalten, dass $\int \mathbb{S} 96$ Abs. 2 AktG-Entwurf und 24 Abs. 3 SEAG-Entwurf von einem internationalen Anwendungswillen getragene Eingriffsnormen darstellen, die grundsätzlich nicht nur auf deutsche Gesellschaften, sondern auch auf Auslandsgesellschaften anwendbar sind.

\section{Sonderanknüpfung der Quotenregelung}

Nachdem gezeigt werden konnte, dass $\int \mathbb{S} 96$ Abs. 2 AktG-Entwurf und 24 Abs. 3 SEAG-Entwurf im Grundsatz international zwingend beabsichtigt sind, soll diese These im Folgenden näher präzisiert und begründet werden.

\section{Reichweite des internationalen Geltungswillens: Nur Quotenregelung, nicht Implementierungs- und Sanktionierungsregime}

Das Gesetz gebietet nicht lediglich die Einhaltung der beschriebenen Geschlechterquote, sondern ordnet darüber hinaus an, dass eine quotenwidrige Wahl oder Entsendung zum Aufsichtsrat nichtig ist. Dabei besteht zwischen beiden Regelungen eine Zweck-Mittel-Relation: Die Nichtigkeitsanordnung ist ein - durchaus drastisches - Instrument, um die Einhaltung der Quotenregelung zu gewährleisten ${ }^{90}$. Gleichwohl sind auch andere Sanktionen denk-

88 Zur diesbezüglichen, aus der Niederlassungsfreiheit abgeleiteten mittelbaren Rechtswahlfreiheit im Internationalen Gesellschaftsrecht WELLER, aaO (Fn. 10), S. 29 ff, $68 \mathrm{ff}$.

$89 \mathrm{Zu}$ den institutionenökonomischen Aspekten dieses Wettbewerbs der Rechtsformen siehe: Thomale, ZEuP 2015, Heft 3 m. w. N. (im Erscheinen).

90 BT-Drucks. $18 / 3784$ v. 20.1.2015, S. 47 formuliert: „Die Nichtigkeitsfolge wirkt ver- 
bar. Die deutsche Aktienrechtordnung kennt etwa als Hauptalternative und Regelfall einer fehlerhaften Aufsichtsratswahl die Anfechtbarkeit nach $\$ 251$ AktG. Weiteres Anschauungsmaterial bietet die Rechtsvergleichung. So kann die Nichtbefolgung von Quotenbestimmungen auch über das Ordnungswidrigkeitsrecht (Geldbußen), das Steuerrecht (Verlust von Steuerprivilegien), das Vergaberecht (Ausschluss des Unternehmens von öffentlichen Aufträgen) oder durch die Versagung der Aufsichtsratsvergütung sanktioniert werden ${ }^{91}$.

Vor diesem Hintergrund stellt sich kollisionsrechtlich die Frage, ob nur die für den Aufsichts- bzw. Verwaltungsrat geltende 30\%- Quotenvorgabe als solche ( $\$ 96$ Abs. 2 Satz 1 AktG-Entwurf und $\$ 24$ Abs. 3 Satz 1 SEAG-Entwurf) oder auch die Nichtigkeitssanktion bei quotenwidriger Besetzung ( $\mathbb{S} 96$ Abs. 2 Satz 6 AktG-Entwurf) internationalen Geltungswillen beinhaltet und dementsprechend auf Auslandsgesellschaften im Fall der Nichtbeachtung der Quotenvorgabe anzuwenden ist. Dies macht einen Blick auf die allgemein im IPR diskutierte Reichweite von Sonderanknüpfungen notwendig.

Im Ausgangspunkt unstreitig ist, dass die Sonderanknüpfung zu einer punktuellen Überlagerung des anwendbaren Rechts durch die sonderangeknüpfte Rechtsnorm führt ${ }^{92}$. Offen bleibt dabei jedoch, ob und inwiefern die Rechtsfolge der Eingriffsnorm ebenfalls dem Eingriffsstatut oder aber der durch die Eingriffsnorm überlagerten lex causae zu entnehmen ist ${ }^{93}$. Für eine Maßgeblichkeit des Eingriffsstatuts für sowohl Tatbestand als auch Rechtsfolge streitet die konsequente Handhabung der Sonderanknüpfung ${ }^{94}$. Nur die Eingriffsnorm könne über ihren Inhalt und ihre direkte Folge (wie z. B. Nichtigkeit) bestimmen ${ }^{95}$. Was sich daraus mittelbar für den Vertrag ergibt (z. B. Rückabwicklung), ergebe sich wiederum aus der lex causae ${ }^{96}$.

Allerdings ist die Anwendung einer Norm in vollem Tatbestands- und Rechtsfolgenumfang nicht denknotwendiges Kennzeichen einer Sonderanknüpfung97. Vielmehr kann man ohne weiteres auch zu einer „gemischten Anwendung" von Tatbestand einerseits und Rechtsfolge andererseits gelangen, indem

haltenssteuernd, weil jede Bank im Aufsichtsrat das Bestreben hat, ihre Plätze zu besetzen."

91 Vgl. rechtsvergleichend zur Quote Teichmann/Langes, Frauenquote in Deutschland und Europa, EWS 2013, 175 ff; WAAs, Geschlechterquoten für die Besetzung der Leitungsgremien von Unternehmen Bewertung der aktuellen Entwürfe aus unionsrechtlicher und rechtsvergleichender Sicht, HSI Schriftenreihe Band 3, 2012; FrancoIs-PonCET/Deilmann, Frauenquote in französischen Aufsichts- und Verwaltungsräten - ist eine Quote auch in Deutschland zulässig?, NGZ 2011, $450 \mathrm{ff}$.

92 SANDrock, AG 2004, 57, 63; Weller, FS Hommelhoff, S. 1275, 1286.

93 von Bar/Mankowski, aaO (Fn. 44), $\$ 4$ Rdn. 111.

94 von Bar/Mankowski, aaO (Fn. 44), $\$ 4$ Rdn. 111.

95 Martiny, aaO (Fn. 45), Rom I-VO, Art. 9 Rdn. 53.

96 Martiny, aaO (Fn. 45), Rom I-VO, Art. 9 Rdn. 54.

97 Schurig, RabelsZ 54 (1990), 217, 241. 
der eingriffsrechtliche Aussagegehalt (hier: 30\%-Quote) in den Normbestand der lex causae eingebettet wird ${ }^{98}$.

Eine solche differenzierende Lösung ist u.E. vorzugswürdig. Für sie spricht erstens, dass zur Qualifikation als Eingriffsnorm jede einzelne Aussage der Norm getrennt auf ihren eingriffsrechtlichen Charakter hin analysiert werden muss ${ }^{99}$. Zweitens soll das Eingriffsrecht das anwendbare Recht nur insoweit verdrängen, als es „unbedingt notwendig ist “ ${ }^{100}$. Der Gedanke des schonendsten Eingriffs findet sich ebenso bei dem mit den Eingriffsnormen verwandten ordre public ${ }^{101}$, der das Ergebnis der Verweisung nur insoweit korrigiert, als es nötig ist, um einen ordre public-Verstoß zu verhindern ${ }^{102}$. Auf dem Gebiet des Europäischen Kollisionsrechts ergibt sich der Grundsatz des schonendsten Eingriffs in die Verweisungsentscheidung drittens aus dem unionsrechtlichen Prinzips des effet utile ${ }^{103}$. Schließlich ist in Erinnerung zu rufen, dass die einseitige Regelung internationaler Sachverhalte durch Eingriffsnormen eine eng umgrenzte Ausnahme vom grundsätzlich bilateralen Kollisionsrechtssystem darstellt.

Vor diesem Hintergrund kann mangels einer ausdrücklichen Regelung oder Aussage im Gesetz und in der Gesetzesbegründung nur die Quotenregelung als sozialpolitischer Kern des Gesetzesvorhabens - mit einem internationalen Anwendungsbefehl versehen werden ${ }^{104}$. Hingegen stellt die Nichtigkeitsrechtsfolge, die im Gesetz kaum begründet wird, eine zwar geeignete, aber keineswegs notwendige Sanktion dar, um die Quotenregelung durchzuset$z^{2}{ }^{105}$. Folglich hat die Nichtigkeitssanktion keinen Eingriffscharakter und ist mithin nicht auf Auslandsgesellschaften anzuwenden. Vielmehr tritt die deutsche Nichtigkeitsanordnung hinter das im jeweiligen Gesellschaftsstatut vorgesehene Sanktionsregime zurück: Kennt etwa ein ausländisches Gesellschaftsrecht nur die Anfechtungslösung für fehlerhafte Bestellungsakte, so ist die quotenwidrige Wahl eines Aufsichtsrats- oder Verwaltungsratsmitglieds nicht nichtig, sondern anfechtbar ${ }^{106}$.

98 Schurig, RabelsZ 54 (1990), 217, $241 \mathrm{ff}$.

99 von Bar/Mankowski, aaO (Fn. 44), $\$ 4$ Rdn. 95.

100 Martiny, aaO (Fn. 45), Rom I-VO, Art. 9 Rdn. 53.

101 Zum Zusammenhang von (negativem) ordre public und (positivem) Eingriffsrecht Weller, aaO (Fn. 9), Einl. Int. GesR Rdn. 431 ff.

102 M. STÜRner, BeckOGK 2015, Rom I, Art. 21 Rdn. 37 (im Erscheinen).

103 M. STÜRner, aaO (Fn. 102), Rom I, Art. 21 Rdn. 37.

104 Zur anerkannten analytischen Vorgehensweise der Eingriffsnormqualifikation siehe von Bar/Mankowski, aaO (Fn. 44), \$4 Rdn. 95: „Dabei ist grundsätzlich jede einzelne Normaussage isoliert zu untersuchen. Eine Norm, ein Paragraph, ein Absatz, ja selbst ein Satz eines Gesetzes kann mehrere Aussagen enthalten, von denen einzelne eingriffsrechtlich, andere nicht-eingriffsrechtlich zu qualifizieren sind.“

105 Vgl. Weller, Stellungnahme für den Familien- und Rechtsausschuss des Deutschen Bundestages zur BT-Drucks. 18/3784, Ausschussdrucksache 18(13)43 k.

$106 \mathrm{Vgl}$. für rechtsvergleichende Beispiele zum Beschlussmängelrecht FLEIScher, Reform- 
Für die weitere Untersuchung ist mithin festzuhalten: Nur die 30\%-Quotenvorgabe als solche ( $\$ 96$ Abs. 2 Satz 1 AktG-Entwurf und $\$ 24$ Abs. 3 Satz 1 SEAG-Entwurf) findet qua Sonderanknüpfung auf Auslandsgesellschaften Anwendung, nicht jedoch die Art und Weise der Bestellung der Aufsichtsratsmitglieder und nicht die Folgen einer quotenwidrigen Besetzung (Nichtigkeitsfolge, $\mathbb{9} 96$ Abs. 2 Satz 6 AktG-Entwurf).

\section{Substitution}

Was „von oben“, aus kollisionsrechtlicher Meta-Perspektive, wie eine unilaterale Eingriffsnorm erscheint, lässt sich „von unten“ auf dem Standpunkt des anwendbaren Sachrechts als Substitution ${ }^{107}$ formulieren. Die kollisionsrechtliche These, der zufolge sich die Quotenregelung auch auf ausländische Gesellschaftsstatute erstreckt, und die sachrechtliche These, nach der das persönliche Tatbestandsmerkmal ,AG' - oder, über den gesetzeseigenen Verweis in $\$ 278$ Abs. 3 AktG das Tatbestandsmerkmal , $\mathrm{KGaA}^{`}$ - durch ausländische Rechtsformen substituiert werden kann, sind bei normlogischer Betrachtung gleichwertig. Deshalb können zur Plausibilisierung der These vom international zwingenden Charakter der Quotenregelung auch solche Sachnormen herangezogen werden, in deren Rahmen das deutsche Recht heute bereits eine Gleichstellung inländischer und ausländischer Gesellschaftsformen vollzieht:

Hier ist zunächst auf die Fälle des identitätswahrenden Hereinformwechsels von einer unionsausländischen in eine deutsche Rechtsform hinzuweisen. Unter Beachtung der Leitentscheidungen des EuGH in Cartesio ${ }^{108}$ und Vale ${ }^{109}$ ist die Rechtsprechung hier dazu übergegangen, die $\$ \mathbb{\$} 1,3,191$ Abs. 1 UmwG unionskonform dahingehend auszulegen, dass auch ausländische Gesellschaften als formwechselnde Rechtsträger anzuerkennen sind, obwohl der Gesetzeswortlaut des Umwandlungsgesetzes nur inländische Gesellschaftsformen nennt ${ }^{110}$. In ähnlicher Weise wird auch die Haftung des Geschäftsführers für Zahlungen nach Insolvenzreife gemäß $\$ 64 \mathrm{GmbHG}$ auf die Geschäftsleiter von Auslandsgesellschaften erstreckt ${ }^{111}$. Ferner sei-so der Gesetzgeber-auch

perspektiven des aktienrechtlichen Beschlussmängelrechts im Lichte der Rechtsvergleichung, AG 2012, $765 \mathrm{ff}$.

107 Näher zur Substitution Mansel, FS W. Lorenz, 1992, 689 ff; Weller, ZGR 2014, 866, $875 \mathrm{ff}$.

108 EuGH Urt. v. 16.12. 2008 - Rs. C-210/06, Slg. 2008, I-9641 - Cartesio.

109 EuGH Urt. v. 12.07.2012 - Rs. C-378/10 - Vale; hierzu Weller/RentsCH, IPRax 2013, 530.

110 OLG Nürnberg, NZG 2014, 349, 350; hierzu L. HüBnER, IRPax 2015, Heft 2.

111 BGH, NZG 2015, 101, 102 (Geltung des $\$ 64$ GmbHG für den Director einer englischen Limited). Vgl. zur Problematik ferner Weller/Schulz, IPRax 2014, 336. 
die inländische Insolvenzverschleppungshaftung , in Insolvenzverfahren über das Vermögen ausländischer Gesellschaften anzuwenden, deren Tätigkeitsschwerpunkt in Deutschland liegt. " 112

Die angeführten Beispiele zeigen nicht nur, dass eine Erstreckung deutscher Sachnormen mit gesellschaftsrechtlichem Gehalt auf Auslandsgesellschaften überhaupt stattfindet. Vielmehr erhellen sie zudem die weitere Voraussetzung, dass die tatbestandlich ersetzte deutsche Gesellschaftsform (AG, GmbH) und die im Wege der überbrückenden Substitution ${ }^{113}$ eingesetzte ausländische $\mathrm{Ge}$ sellschaftsform funktionell gleichwertig sein müssen. So wurde etwa der Begriff der „Kapitalgesellschaft“ i. S. d. $\iint 3$ Abs. 1 Nr. 2, 191 Abs. 1 Nr. 2 UmwG mit Hilfe des in Art. 1 der Publizitätsrichtlinie ${ }^{114}$ enthaltenen Katalogs für ausländische Kapitalgesellschaften verallgemeinert ${ }^{115}$. Entsprechend wurde die englische Limited der deutschen $\mathrm{GmbH}$ mit der Begründung gleichgestellt, dass beide Gesellschaftsformen den Prinzipien der beschränkten Gesellschafterhaftung und der Fremdorganschaft folgten und daher ein typisches Insolvenzverschleppungsrisiko mit sich brächten ${ }^{116}$. Methodisch betrachtet findet hier eine normfunktionale Gesellschaftsrechtsvergleichung statt. Unter den Auspizien der nationalen Sachnorm, deren vorgesehene inländische Rechtsform zu ersetzen ist, sind beide Rechtsformen wertend miteinander zu vergleichen ${ }^{117}$. Für die Quotenregelung der $\$ \$ 96$ Abs. 2 AktG-Entwurf und 24 Abs. 3 SEAG-Entwurf bietet sich in dieser Hinsicht der Begriff der Publikumsgesellschaft als Gattung der deutschen AG und KGaA an. Dieser wird weiter charakterisiert durch den in Bezug genommenen $\$ 1$ Abs. 1 Nr. 2 MitbestG, welcher eine Regelbeschäftigung von mehr als 2000 Arbeitnehmern verlangt.

Somit erstreckt sich die Quotenregelung grundsätzlich auf ausländische börsennotierte Publikumsgesellschaften mit einer Regelbeschäftigung von mehr als 2000 Arbeitnehmern.

\section{Inlandsbezug}

Um den eingriffsrechtlichen Bruch mit der kollisionsnormgemäßen Ausgangslage zu beschränken und damit gleichzeitig zu rechtfertigen, ist für die Anwendbarkeit einer inländischen Eingriffsnorm im konkreten Fall ein In-

112 BT-Drucks. 16/6140, 47. So auch KG, NZG 2010, 71; Weller, FS Ganter, 2010, 339 ff.

113 Begriff nach: von Bar/Mankowski, aaO (Fn. 44), $\$ 7$ Rn. 241.

114 RL 2009/101/EG.

115 OLG Nürnberg, NZG 2014, 349, 350.

116 BGH, NZG 2015, 101, 102.

117 Zu diesem Vorgang im Rahmen der Substitution allgemein von BAR/MANKowsKI, aaO (Fn. 44), \$7 Rn. 243. Die übliche Terminologie nennt hier den ersetzten Begriff des eigenen Rechts ,Systembegriff‘ und den eingesetzten Begriff ,Substitutionsbegriff‘. 
landsbezug des Sachverhalts erforderlich ${ }^{118}$. Diese Voraussetzung leitet sich aus der institutionellen Verwandtschaft des inländischen Eingriffsrechts mit dem nationalen ordre public-Vorbehalt ${ }^{119}$ sowie aus der Tatsache ab, dass ausländische Eingriffsnormen ebenso allein bei einer „engen Verbindung“ zum Sachverhalt zu berücksichtigen sind ${ }^{120}$ : Ohne einen hinreichend unmittelbaren Wirkungszusammenhang mit dem Inland muss eine inländische Norm nicht durchgesetzt werden, sondern hat sich vielmehr dem kollisionsrechtlich vorgesehenen ausländischen Sachrecht unterzuordnen ${ }^{121}$. Freilich wird im sachrechtlich operierenden Institut der Substitution das Erfordernis eines Inlandsbezugs, soweit ersichtlich, noch nicht diskutiert. Dies resultiert jedoch aus einer offensichtlichen Anschauungslücke. Bislang wurde die Substitution lediglich dazu eingesetzt, über die kollisionsrechtliche Verweisung hinaus eine zusätzliche sachrechtliche Öffnung der nationalen Rechtsordnung für ausländisches Recht herbeizuführen - nicht hingegen, um umgekehrt nationales Recht auf ausländische Statute zu erstrecken und diese damit zu beschränken $^{122}$. Zwar ist der substituierende modus operandi vorliegend derselbe: Ein inländischer Systembegriff (, $\mathrm{AG}^{\prime}$ oder , $\mathrm{KGaA}^{\prime}$ ) wird durch einen funktional äquivalenten Substitutionsbegriff ersetzt. Die Wirkung ist jedoch eine entgegensetzte, da auf diese Weise der Anwendungsbereich inländischen Sachrechts erweitert werden soll. Es handelt sich um eine expansive Form der Substitution. Diese muss demselben Rechtfertigungsdruck unterworfen werden, dem jeder Eingriff in die kollisionsrechtlich bestimmte lex causae standzuhalten hat. Somit ist vorliegend ebenfalls unter dem sachrechtlichen Aspekt

118 Kropholler, aaO (Fn. 69), 498 f; Martiny, aaO (Fn. 45), Art. 9 Rom I-VO Rdn. 122.

119 Näher Weller, aaO (Fn. 9), Einl. Int. GesR Rdn. 431 ff.

120 Ähnlich: Kropholler, aaO (Fn. 69), 246, 498, 506 ff. Eine enge Verbindung ausländischer Eingriffsnormen verlangte noch ausdrücklich $\$ 7$ EVÜ, der weiterhin eine wichtige Rechtserkenntnisquelle des aktuell im internationalen Vertragsrecht geltenden Art. 9 Abs. 3 Rom I-VO darstellt. Zur Ideengeschichte siehe: Thomale, IPRax 2013, $375 \mathrm{ff}$.

121 Vgl. Martiny, aaO (Fn. 45), Art. 9 Rom I-VO Rdn. 109.

122 Die Substitution ist mithin dem Grunde nach einseitig wirksam. Im Gesellschaftsrecht siehe etwa die ausdrückliche Substitutionsoffenheit des $\$ 3$ Abs. $2 \mathrm{AktG}$, der laut seiner Gesetzesbegründung in BT-Drucks. 13/9712, S. 12 re. Sp. „auch [die Notierung] an vergleichbaren Börsen im Ausland erfasst." sowie die grundsätzliche Öffnung des $\$ 6$ GmbHG für im Ausland wohnhafte Geschäftsführer, dazu OLG Köln, IPRax 2000, 130. Diese Ansicht bestreiten auf den ersten Blick von BAR/Mankowski, aaO (Fn. 44), $\$ 7$ Rn. 242 mit dem Hinweis darauf, die Substitution komme „selbst dann [in Betracht], wenn es darum geht, ob ein ausländisches Ausgangsrecht durch Inlandsgestaltungen ausgefüllt werden kann." Dabei beziehen sie sich auf Fälle, in denen ausländische Urkundsbeamte auf deutschem Staatsgebiet Beurkundungen vornehmen und diese sodann gleichwohlunter den ausländischen Systembegriff der Beurkundung gefasst werden, vgl. Renм, RabelsZ 64 (2000), 104, 108 ff. Doch dehnt auch diese Spielart der Substitution die Anwendbarkeit ausländischen Rechts aus und steht damit den üblichen anerkannten Fallgruppen wesentlich gleich. 
der expansiven Substitution ein enge Verbindung in Form eines hinreichenden Inlandsbezugs zu verlangen.

Mit dem herausgearbeiteten Kriterium des hinreichenden Inlandsbezugs ist die entscheidende Wertungsfrage zwar gestellt, aber nicht beantwortet. Unter welchen Voraussetzung weist eine ausländische börsennotierte Publikumsgesellschaften mit einer Regelbeschäftigung von mehr als 2000 Arbeitnehmern einen Bezug zur deutschen Rechtsordnung auf, der hinreicht, um die Quotenregelung auf sie zu erstrecken? Dies kann nur vom sozialen Gestaltungswillen der Quotenregelung her bestimmt werden. Dabei ist von entscheidender Bedeutung, dass die Quotenregelung mitsamt ihrer gleichstellungspolitischen Zielsetzung auf den Wirtschafts- und Sozialstandort Deutschland fixiert ist. Mithin ist eine erste Voraussetzung für einen hinreichenden Inlandsbezug darin zu erblicken, dass die Auslandsgesellschaft ihren Verwaltungssitz in Deutschland hat. Dadurch wird der unmittelbare Regelungswille des Gesetzes international übersetzt, in Deutschland wirkende Aufsichts- und Verwaltungsräte mit einer Mindestgeschlechterdiversität auszustatten. Die Quotenregelung sieht darin jedoch keinen Selbstzweck, sondern zielt vielmehr auf einen Trickle-down-Effekt ab, der von der Unternehmensspitze ausgehend die weiteren Managementund Angestelltenebenen erfassen und zu einer neuen Unternehmenskultur in Deutschland führen soll ${ }^{123}$. Diesem eigentlichen Gestaltungsanspruch der Quotenregelung kann international- privatrechtlich dadurch entsprochen werden, dass sich nicht nur die Unternehmensführung selbst, sondern zweitens auch die 2000 Mindestregelbeschäftigten in Deutschland befinden müssen, um einen hinreichenden Inlandsbezug zu gewährleisten. Hingegen setzt das Gesetz zwar eine Börsennotierung der betroffenen Gesellschaft voraus, stellt jedoch keinen kapitalmarktbezogenen Schutz- oder Gestaltungszusammenhang zwischen Börsennotierung und Quotenregelung her. Eine Notierung an einer deutschen Börse ist daher nicht zwingend erforderlich ${ }^{124}$.

Somit liegt ein genügender Inlandsbezug vor, wenn die Auslandsgesellschaft ihren Verwaltungssitz in Deutschland hat und dort regelmäßig mehr als 2000 Arbeitnehmer beschäftigt.

\section{Europarechtskonformität}

$\mathrm{Zu}$ prüfen ist schließlich, ob die über das Kollisionsrecht erfolgende eingriffsrechtliche Durchsetzung der Quotenregelung gegenüber Auslandsgesellschaften auch unionsrechtskonform ist. Ausgangspunkt ist dabei zunächst die Er-

123 Siehe oben unter V.3.

124 Für dieses Ergebnis spricht ferner, dass es auch für die Legaldefinition der „Börsennotierung “ in $\$ 3$ Abs. 2 AktG unerheblich ist, ob die Gesellschaft im In- oder Ausland börsennotiert ist. 
kenntnis, dass sich das dogmatische Gerüst der Eingriffsnormen mit der europäischen Kollisionsrechtsharmonisierung nur unmerklich verändert hat ${ }^{125}$. Über Art. 9 Abs. 2 Rom I-VO und Art. 16 Rom II-VO wird nämlich mitgliedstaatliches ordnungspolitisches Recht vom Anwendungsbereich des EU-Kollisionsrechts ausgenommen ${ }^{126}$. Obwohl der Europäische Gerichtshof in der Vergangenheit Leitlinien für einen autonom-unionsrechtlichen Begriff ordnungspolitischen Rechts entwickelt hat ${ }^{127}$, liegt die Einschätzungsprärogative darüber, wie sich eine Eingriffsnorm definiert, nach wie vor bei den Mitgliedstaaten $^{128}$. Aus dem sekundärrechtlichen Harmonisierungsdefizit folgt andererseits aber nicht, dass die Mitgliedstaaten bei der Anwendung ihrer nationalen zwingenden Vorschriften nicht an die Vorgaben des Unionsrechts gebunden sind. Insbesondere kann die Durchsetzung von Eingriffsrecht obgleich durch das sekundärrechtliche EU-Kollisionsrecht „abgesegnet“ gegen die primärrechtlichen Grundfreiheiten verstoßen.

\section{Gewährleistungsbereich der Niederlassungsfreiheit}

Die Niederlassungsfreiheit schützt sowohl die Aufnahme und Ausübung einer selbstständigen Erwerbstätigkeit als auch die grenzüberschreitende Gründung und Leitung von Unternehmen ${ }^{129}$. Im Gesellschaftskollisionsrecht setzt sich diese sachrechtliche Verbürgung in zwei Facetten um. Erstens in Form einer grenzüberschreitenden Wahlfreiheit der Unternehmensgründer in Bezug auf das Gesellschaftsstatut ${ }^{130}$; zweitens durch die Überlagerung der in Kontinentaleuropa vormals etablierten Sitz ${ }^{131}$ - durch die Gründungstheorie $^{132}$. Entsprechend ist für das Gesellschaftsstatut nicht mehr der Verwal-

125 Johannes Fetsch, Eingriffsnormen und EG-Vertrag, 2002, $237 \mathrm{ff}$.

126 Dazu RenTsCh, Krisenbewältigung durch konstitutionalisiertes Kollisionsrecht, oder: Eingriffsrecht als integraler Bestandteil des europäischen IPR, in: Bauerschmidt/Fassbender/Müller/Siehr/Unseld, Konstitutionalisierung in Zeiten globaler Krisen, 2015, $255,265 \mathrm{f}$.

127 EuGH, 23. 11.1999-C-369/96, Arblade und Leloup: „(...) unter diesem Begriff [sind] nationale Vorschriften zu verstehen, deren Einhaltung als so entscheidend für die Wahrung der politischen, sozialen oder wirtschaftlichen Organisation des betreffenden Mitgliedstaats angesehen wird, dass ibre Beachtung für alle Personen, die sich im nationalen Hoheitsgebiet dieses Mitgliedstaats befinden, und für jedes dort lokalisierte Rechtsverbältnis vorgeschrieben ist." (Herv. d. VERF.)

128 Vgl. EuGH, Urteil v. 17. 10. 2013 - C-184/12, Rdn. 50 - Unamar m. Anm. Schilling, ZEuP 2014, 845.

129 Forsthoff, in: Grabitz/Hilf/Nettesheim, Das Recht der Europäischen Union, 54. Ergänzungslieferung 2014, Art. 49 AEUV Rdn. 16.

130 Weller, aaO (Fn. 10).

131 Zur historischen Entwicklung Kindler, aaO (Fn. 58), IntGesR Rdn. 420; Trautrims, ZHR 176 (2012), $435 \mathrm{ff}$.

132 Weller, aaO (Fn. 9), Int. GesR Rdn. 333. 
tungs-, sondern der Satzungssitz einer Gesellschaft entscheidend ${ }^{133}$. Auslandsgesellschaften mit Satzungssitz in einem anderen EU-Mitgliedstaat sollen also in Deutschland unabhängig vom Kanon der hiesigen Gesellschaftsformen unter ihrem frei erwählten Satzungsstatut einer selbstständigen Tätigkeit nachgehen können.

\section{Beschränkung der Niederlassungsfreiheit}

Die so geschaffene Rechtsformwahlfreiheit wird durch jeden Übergriff des deutschen Sachrechts in das ausländische Gesellschaftsstatut i. S. d. Dassonville-Formel beschränkt. Ein solcher findet statt, indem die $\$ \mathbb{S} 96$ Abs. 2 AktG-Entwurf und 24 Abs. 3 SEAG-Entwurf auf Auslandsgesellschaften erstreckt und so die auf Grundlage der Art. 49, 54 AEUV grundsätzlich abschließenden ausländischen Organisationsnormen überlagert oder verändert werden. Daher muss die Quotenregelung nach der sog. Cassis-Formel vier Voraussetzungen erfüllen, um trotz der Beschränkung auf Rechtfertigungsebene den Anforderungen des EU-Primärrechts zu genügen ${ }^{134}$ : Sie muss erstens unterschiedslos gelten, zweitens zwingende Gründe des Allgemeinwohls bedienen und diese drittens in geeigneter und viertens in erforderlicher Weise umsetzen $^{135}$.

\section{Keck-Ausnabme: Quotenregelung als bloße Tätigkeitsausübungsmodalität?}

Die Notwendigkeit einer Rechtfertigung nach dem Schrankenregime der Niederlassungsfreiheit wird andererseits obsolet, wenn man zum Ergebnis kommt, dass die Quotenregelung nicht den Zugang einer Auslandsgesellschaft zum deutschen Markt regelt, sondern als Tätigkeitsausübungsregel i.S.d. Keck-Formel lediglich den Gebrauch der Zuzugsfreiheit nach erfolgtem Marktzugang reguliert ${ }^{136}$.

Das Centros-Urteil des EuGH deutet implizit an, dass sich die in Keck zur Warenverkehrsfreiheit erfolgte Schutzbereichskonkretisierung auch auf die von der Niederlassungsfreiheit geschützte Unternehmensmobilität übertra-

133 BGH Urt. v. 13.3. 2003 - VII ZR 370/98, IPRax 2003, 344 = NJW 2003, 1461; dazu Weller, IPRax 2003, 324; Leible/Hoffmann, ZIP 2003, 925; Eidenmüller, JZ 2003, 526.

134 Für die Niederlassungsfreiheit EuGH, Urt. vom 30.11.1995, Rs. C-55/94, Slg. 1995, I-4165 - Gebhard, Rdn. 37.

135 Siehe infra unter 4.-6.

136 EuGH, Urt. vom 24.11.1993, Rs. C-267/91 und C-268/91, Slg. 1993, I-6097 - Keck und Mithouard = NJW 1994, 121. 
gen lassen soll137. Dafür spricht, dass die Keck-Formel nur in der Anfangsphase als vertriebsbezogene Bereichsausnahme verstanden wurde, die für Verkaufsmodalitäten ein anderes Rechtfertigungsregime (Unterschiedslosigkeit) aufstellt als für Marktzugangsregelungen ${ }^{138}$, mittlerweile aber einen bedeutenden Funktionswandel hin zu einer verallgemeinerungsfähigen Bereichsausnahme vom Gewährleistungsbereich der Grundfreiheiten durchlaufen hat ${ }^{139}$. Die Keck-Formel liest sich aktuell als Teil eines drei-schrittigen Prüfungsschemas, wonach für Maßnahmen, die (1) de lege unterschiedslos für Inund Ausländer gelten, (2) de facto Ausländer auch faktisch nicht mehr beeinträchtigen als Inländer und (3) den Gebrauch einer Grundfreiheit lediglich konditionieren, aber weder in der Zielsetzung noch im Ergebnis verbieten, keine Rechtfertigungspflicht gilt ${ }^{140}$.

Die $\int \mathbb{S} 96$ Abs. 2 AktG-Entwurf und 24 Abs. 3 SEAG-Entwurf belasten börsennotierte Auslandsgesellschaften jedenfalls im Zeitpunkt ihres Inkrafttretens unter keinem Aspekt stärker als Inlandsgesellschaften. Unabhängig vom anwendbaren Gesellschaftsstatut entsteht nämlich für alle in Deutschland vom sachlichen Anwendungsbereich der Quote umfassten Unternehmen die Pflicht, die Besetzung ihrer Aufsichts- oder Verwaltungsräte entsprechend der Quotenvorgabe neu zu justieren. Der Eingriff in die gesellschaftliche Regelungsautonomie ist also für In- und Auslandsgesellschaften gleich schwer.

Weiterhin unterscheidet sich die Geschlechterquote vom ansonsten parallel gelagerten Fall der Mitbestimmung durch ihre fehlende unmittelbare wirtschaftspolitische Konnotation. Die Vorschriften zur Binnenorganisation von

137 Weller, FS Hommelhoff, S. 1275, 1293 m. w. N.

138 EuGH, Urt. vom 24.11.1993, Rs. C-267/91 und C-268/91= NJW 1994, 121 Rdn. 16; Brigola, EuZW 2012, 248.

139 Anschaulich als „Metamorphose“ bezeichnet wird diese Entwicklung bei BRIGOLA, EuZW 2012, 248 f. Bereits in den Schlussanträgen zur Rechtssache EuGH, Urt. v. 14. 9. 2006, C-158/04 und C-159/04, Slg. 2006, I-8135, Alfa Vita Vassilopoulos AE, Slg. 2006, I-8135; dazu REICH, EuZW 2006, 304, hatte sich Generalanwalt Maduro dafür ausgesprochen, die Keck-Formel von einem formal-anlassbezogenen zu einem rein wirkungsakzessorischen, materiellen Test weiterzuentwickeln, vgl. dazu MADURO, Schlussanträge v. 30.3.2006, BeckRS 2012, 80538 Rdn. 42-45; 48-51. Der Gerichtshof war diesem Vorschlag zunächst nur verhalten gefolgt, EuGH, Urt. v. 14. 9. 2006, C-158/04 und C-159/04, Rdn. 19 aE; Brigola, EuZW 2012, 249, nahm ihn aber in der Entscheidung EuGH, Urt. v. 4. 6. 2009, Rs. C-142/05, Slg. 2009, I-4273, Mickelsson/Roos, Slg. 2009, I-4273 auf. Vgl. dazu auch die Schlussanträge der Generalanwältin Кокотт v. 14.12.2006, BeckRS 2012, 80539. Rechtfertigungsbedürftig sind also nur solche Regelungen, die unabhängig von ihrem Regelungsziel die Unternehmensmobilität derart intensiv beeinträchtigen, dass ihr Gebrauch faktisch vereitelt wird. Dagegen gilt eine Hinnahmepflicht für die Folgen eines an sich zulässigen Gebrauchs nationaler Regulierungsautonomie, sofern er In- und Ausländer rechtlich und faktisch gleichermaßen belastet.

140 Schlussanträge der Generalanwältin Кокотт v. 14.12.2006, BeckRS 2012, 80539. 
Kapitalgesellschaften sind nämlich nur deshalb Teil der Reformdiskussion, weil sich der Gesetzgeber der dort zur Verfügung stehenden Mechanismen bedient, um eine gesellschaftsrechtsexterne Werteentscheidung durchzusetzen. Zwar kann man das Anliegen, führende Gremien geschlechterparitätisch zu besetzen, wie supra bereits erwähnt wurde ${ }^{141}$, nicht nur sozialpolitisch, sondern auch ökonomisch rechtfertigen, da die regulierte Besetzung der Führungsebene eine Verbesserung des Arbeitsklimas und eine positive Veränderung der Unternehmenskultur in Deutschland erwarten lässt ${ }^{142}$. Die unmittelbare Motivation für die Geschlechterquote liegt aber, wie neben der Präambel der EU-Gründungsverträge ${ }^{143}$ auch die Art. 2 EUV, Art. 3 Abs. 3 EUV, Art. 10 AEUV und schließlich Art. 157 Abs. 1 AEUV zeigen, darin, den europäischen Wertekonsens zur Gleichheit der Geschlechter und das damit verbundene sozialpolitische Anliegen arbeitsrechtlicher Gleichberechtigung durchzusetzen. Die Quote stellt im Ergebnis also keine genuin gesellschaftsrechtliche Regelung dar, sondern konkretisiert eine viel allgemeinere Marktteilnahmeregel, wonach Führungspositionen ungeachtet ihrer institutionellen Einhegung geschlechterparitätisch zu besetzen sind.

Unter dieser Prämisse kann es sich bei der Geschlechterquote nicht um eine Marktzugangsregel für Gesellschaften handeln; vielmehr wird mit ihr eine Tätigkeitsausübungsregel i.S.v. Keck aufgestellt, die als Bereichsausnahme von der Niederlassungsfreiheit keine Rechtfertigungslast auslöst.

Indes: Ein Blick auf den Ansatzpunkt und den Regelungsgegenstand der Quotenregelung relativiert dieses Ergebnis. Durch eine Konditionierung der Wahlregeln für den Aufsichtsrat nach den $\$ \mathbb{\$} 96$ Abs. 2 AktG-Entwurf und 24 Abs. 3 SEAG-Entwurf wird unmittelbar in die Gesellschaftsverfassung eingegriffen. Die zuzugswilligen Unternehmen werden entsprechend mit einer zusätzlichen Regel zur Binnenorganisation belastet ${ }^{144}$.

Auch nach dieser zweiten Hypothese ist ein Eingriff in die Niederlassungsfreiheit allerdings jedenfalls dann gerechtfertigt, wenn er einen zwingenden Allgemeinwohlbelang verfolgt und diesen geeignet und erforderlich umsetzt.

141 Vgl. supra unter V.3.b).

142 BT-Drucks. 18/3784, S. 55.

143 Dazu Grupp, in: Bergmann, Handlexikon der Europäischen Union, 4. Aufl., 2011, Stichwort „Gleichstellungspolitik“.

144 So bereits für die Mitbestimmung Weller, FS Hommelhoff, S. 1275, 1293. Hinzu kommt, dass sich die tatsächlichen Auswirkungen der Quotenregelung auf die Unternehmensmobilität im Voraus nur schwer bestimmen lassen. Ob zuzugswillige Auslandsgesellschaften durch die Organisationsvorgabe über die Maßen belastet werden, hängt nämlich nicht zuletzt davon ab, wie mit Unternehmen umgegangen wird, deren Gründungsstatut ebenfalls eine Quotenregelung vorsieht und den Vorgaben des Gesetzes damit zwar nicht im Hinblick auf die konkret festgesetzte 30\%-Quote, wohl aber in der Zielsetzung entspricht. 
Dass es sich bei der Geschlechtergleichheit um einen Allgemeinwohlbelang handelt, ergibt sich aus zahlreichen Vorschriften des Unionsrechts, die ein identisches Ziel verfolgen. Allerdings lässt sich aus der Parallelität der Regelungsziele kein Rechtfertigungsautomatismus am Maßstab der Grundfreiheiten entwickeln. Im Gegenteil muss das Gesetz einen legitimen Allgemeinwoblbelang i.S.d. Cassis-Formel verfolgen (unter 4.). Um das Ziel der Geschlechtergleichheit in geeigneter Weise umsetzen zu können, darf die Quotenregelung das in Art. 157 Abs. 3, 4 AEUV festgelegte Prinzip der arbeitsrechtlichen Geschlechtergleichheit ${ }^{145}$ nicht verletzen. Letzteres ist der Fall, wenn das Gesetz die Voraussetzungen des Art. 157 Abs. 4 AEUV erfüllt, der spezifische Besserstellungen des unterrepräsentierten Geschlechts gestattet. Ferner muss das Gesetz die Vorgaben des Kohärenzgebots erfüllen (unter 5.). Schließlich muss das Kriterium der Erforderlichkeit gegeben sein (unter 6.).

\section{Zwingender Grund des Allgemeinwobls}

\section{a) Gleichstellung als Unionspolitik}

Das Anliegen, die vollständige Gleichstellung von Männern und Frauen in Beruf und Gesellschaft durchzusetzen, ist im Unionsprimärrecht ebenso prominent vertreten wie im deutschen Verfassungsrecht (Art. 3 Abs. 2 GG). Die Art. 2, 3 EUV, 10 AEUV heben das Bekenntnis zur Förderung der Geschlechtergleichheit in den Rang eines gemeinsamen, unionsrechtlichen Wertes. Art. 157 Abs. 4 AEUV gesteht den Mitgliedstaaten darüber hinaus spezifische Begünstigungen von Frauen im Arbeitsleben zu, soweit diese das Ziel verfolgen, bestehende Benachteiligungen auszugleichen. Eine identische Bestimmung ist mittlerweile in Art. 23 Abs. 2 EuGrCh enthalten ${ }^{146}$.

\section{b) Maßstabswabl}

Nur der Gleichstellungsauftrag des Art. 157 AEUV spricht aber ein rechtfertigendes Eingriffsmandat für die Mitgliedstaaten aus ${ }^{147}$. Art. $23 \mathrm{EuGrCh}$ gilt

145 Krebber, in: Calliess/Ruffert, EUV/AEUV, 4. Aufl., 2011, Art. 157 AEUV Rdn. 75 m.w. N.

146 Zum Verhältnis von Art. 23 EuGrCh und Art.157 AEUV vgl. Coen, in: Lenz/Borchardt, EU-Verträge, 6. Aufl., 2013, Art. 157 AEUV Rdn. 3; Schwarze, EUV/AEUV, Art. 157 AEUV Rdn. 1. Zu den Implikationen der Norm für die verfassungsrechtliche Zulässigkeit der Frauenquote vgl. WieLANDT, Stellungnahme zur Vorbereitung der öffentlichen Anhörung des Ausschusses für Familie, Senioren, Frauen und Jugend des Deutschen Bundestages am 23. Februar 2014 zur BT-Drucks. 18/3784, 2 f.

147 Krebber, aaO (Fn. 145), Art. 157 AEUV Rdn. 74; Starck, JZ 2000, 670 (671). 
demgegenüber primär für die Unionsorgane ${ }^{148}$. Für die Mitgliedstaaten ist die Vorschrift „(...) ausschließlich bei der Durchführung des Rechts der Union“ (Art. 51 Abs. $1 \mathrm{EuGrCh}$ ) verbindlich ${ }^{149}$. Die Bundesrepublik könnte die Grundrechtecharta also nur dann rechtfertigend gegen Eingriffe in die Grundfreiheiten anführen, wenn sie funktional im Rahmen der Verbandskompetenz der Union handelt ${ }^{150}$. Unabhängig davon, wie weit der Begriff der „Durchführung" in Art. 51 Abs. 2 EuGrCH verstanden wird ${ }^{151}$, steht vorliegend genau der umgekehrte Fall zur Diskussion, da die Bundesrepublik eigenständig legislative Maßnahmen ergreift ${ }^{152}$. Der unionsprimärrechtliche Maßstab für die Zulässigkeit der Quotenregelung bestimmt sich also ausschließlich nach Art. 157 Abs. 4 AEUV.

\section{Geeignetheit der Quote: Kein Verstoß gegen Art. 157 Abs. 3, 4 AEUV}

\section{a) Geschlechterquote als Ungleichbehandlung}

Die positive Diskriminierung ${ }^{153}$ zugunsten weiblicher Kandidierender bei der Besetzung von Aufsichtsräten wirkt sich, wenn überhaupt, zwar nur mittelbar auf die Frage der Entgeltgleichheit (Art. 157 Abs. 1 AEUV) aus, tangiert aber den in Art. 157 Abs.3, 4 AEUV implizit festgelegten arbeitsrechtlichen Gleichbehandlungsgrundsatz ${ }^{154}$. Obschon das Gesetz sowohl die männliche als auch die weibliche Repräsentation in Aufsichtsräten durch eine entsprechende Quote adressiert, wirkt sich die Regelung in der praktischen Anwendung wohl ausschließlich auf die derzeit unterrepräsentierten Bewerberinnen

148 Kotzur, in: Geiger/Khan, EUV/AEUV Kommentar, 5. Aufl., 2010, Einf. GR-Charta Rdn. 10.

149 Kotzur, aaO (Fn. 148), Einf. GR-Charta Rdn. 10. Zum weiten Verständnis dieses Kriteriums EuGH, Urt. v. 26.2.2013, Rs. C 617/10, Åkerberg Fransson, Rdn. 45 f = EuZW 2013, 302.

150 Zur EU-kompetenzverstärkenden Wirkung der EuGrCh insbesondere im Bereich der Antidiskriminierung Jarass, Zum Verhältnis von Grundrechtecharta und sonstigem Recht, EuR 2013, 29, 32.

151 Für ein enges Verständnis der Durchführung noch Oppermann/Classen/NetTesHEIM, Europarecht, $\$ 18 \mathrm{Rdn} .22$.

152 Nur am Rande sei daher erwähnt, dass der Gleichstellungsauftrag des Art. 23 EuGrCh in seiner Umsetzung nicht nur mit den Grundfreiheiten des AEUV, sondern auch mit anderen Verbürgungen der EuGrCH im Verhältnis praktischer Konkordanz stehen muss. Die Quotenregelung gerät insofern jedenfalls mit dem Recht der EU-Auslandsgesellschaften auf Unternehmerische Freiheit (Art. 16 EuGrCh) in Konflikt. Analog zur Mitbestimmungsproblematik scheint darüber hinaus auch ein Eingriff in die Eigentumsgarantie der betroffenen Unternehmen (Art. $17 \mathrm{EuGrCH}$ ) nicht ausgeschlossen.

153 Dazu Krebber, aaO (Fn. 145), Art. 157 AEUV Rdn. 76 f.

154 Krebber, aaO (Fn. 145) Art. 157 AEUV Rdn. 75 m. w. N. 
begünstigend aus ${ }^{155}$. Für die männlichen Kandidaten ist mit der Regelung dagegen eine bedeutende Schlechterstellung verbunden, da ihr geschlechterspezifischer Bezugs- und Bewerbungsrahmen im Aufsichtsrat von bislang $100 \%$ auf höchstens $60 \%$ herabsinkt ${ }^{156}$.

\section{b) Geschlechterquote als spezifische Besserstellung (Art.157 Abs. 4 AEUV)}

Ebenso wie das deutsche Verfassungsrecht (Art. 3 Abs. 2 GG) erkennt aber auch das Unionsrecht an, dass die Herstellung faktischer Geschlechtergleichheit regelmäßig nicht ohne eine bereichsspezifische oder temporäre rechtliche Besserstellung des unterrepräsentierten Geschlechts möglich ist ${ }^{157}$. Die Mitgliedstaaten erhalten daher durch Art. 157 Abs. 4 AEUV das Recht, spezifische Besserstellungsmaßnahmen zur Herstellung (faktischer) Gleichheit der Geschlechter zu ergreifen. Ein identischer Regelungsauftrag zur positiven Diskriminierung ist auch in Art. 2 Abs. 4 der Antidiskriminierungsrichtlinie enthalten ${ }^{158}$. Jedenfalls für den Zugang zu bestimmten Berufsgruppen in der Privatwirtschaft wurde pauschalen Quotenregelungen bislang allerdings die Rechtfertigung versagt ${ }^{159}$. Das sollte insbesondere gelten, wenn Bewerberinnen gegenüber besser qualifizierten männlichen Bewerbern den Vorzug erhalten sollten ${ }^{160}$. Die Quotenregelung des Gesetzes kann die Rechtfertigungslast aber aus mehreren Gründen meistern. Zum einen gleicht sie in der Sache der Rechtslage im Urteil Marschall ${ }^{161}$, das eine bevorzugte Einstellung von Bewerberinnen unter dem Vorbehalt gleicher Eignung für unionsrechtskonform erklärte ${ }^{162}$. Zum anderen lässt sich mittlerweile auch in der Gesetzgebung der Union eine eindeutige Präferenz für die Umsetzung der Gleichstellungsidee durch Quotenbestimmungen erkennen. Das hier diskutierte Gesetz findet nämlich sowohl in seiner Zielsetzung als auch in seinem Inhalt Entsprechung in einem Richtlinienentwurf der Europäischen Kommission aus dem Jahr

155 Vgl. auch Krebber, aaO (Fn. 145) Art. 157 AEUV Rdn. 76 f.

156 Parallel im Kontext des Art. 3 Abs. 3 GG Windthorst, Stellungnahme zum Gesetzentwurf der Bundesregierung für die gleichberechtigte Teilhabe von Frauen und Männern an Führungspositionen in der Privatwirtschaft und im öffentlichen Dienst vom 20. 01.2015, BT-Drucks. 18/3784, 8.

157 Zum Politikwechsel von der Gleichberechtigung zur Gleichstellung und dem damit verbundenen Gender-Mainstreaming Coen, aaO (Fn. 146), Art. 157 AEUV Rdn. 65.

158 Vgl. bereits Empfehlung 84/635/EWG zur Förderung positiver Maßnahmen für Frauen vom 13.12.1984, ABl. 1985 L 331/34, dazu Coen, aaO (Fn. 146), Art. 157 AEUV Rdn. 47.

159 EuGH, Urt. v. 17.10.1995 - C-450/93, Kalanke = ZIP 1995, 1692.

160 EuGH, Urt. v. 6. 7. 2000 - C-407/98, Slg. 2000, I-2267, Abrahamsson, Anderson/Fogelquist.

161 EuGH, Urt. v. 11.11.1997 - C-409/95, Marschall.

162 EuGH, Urt. v. 11.11.1997 - C-409/95, Marschall, Rdn. 37. 
2012 $2^{163}$, der am 20.11.2013 vom Europäischen Parlament beschlossen wur$\mathrm{de}^{164}$ und in zweiter Lesung im Rat diskutiert wurde ${ }^{165}$. Ebenso wie das dazugehörige Aktionsprogramm ${ }^{166}$ möchte das Gesetz in der vom Parlament beschlossenen Version die ausgeglichene Repräsentation beider Geschlechter in Führungspositionen durch eine verbindliche Quotenregelung in börsennotierten Unternehmen durchsetzen ${ }^{167}$. Schon allein dieser Parallelität wegen wird man davon ausgehen können, dass das Gesetz jedenfalls im Hinblick auf den Umsetzungsmodus - die starre Geschlechterquote - den Anforderungen des Art. 157 Abs. 4 AEUV genügt.

\section{c) Geschlechterquote in börsennotierten Unternehmen als kobärente (geeignete) Schrankenregelung?}

Andererseits lässt sich die Geeignetheit der Quotenregelung unter Kohärenzgesichtspunkten bezweifeln. Um das Anliegen geschlechterparitätischer Repräsentation im Arbeitsleben allgemein und umfassend umzusetzen, ist die vom Bundestag beschlossene Regelung prima facie zu selektiv. Zunächst gilt die Quote aus der Vielzahl der am Wirtschaftsleben beteiligten Personenmehrheiten ausschließlich für Kapitalgesellschaften, während Personengesellschaften und Stiftungen weiterhin keiner Regulierung unterworfen sind. Innerhalb der Kapitalgesellschaften werden dann in einem weiteren Schritt nur solche Unternehmen der Quotenregel unterworfen, die börsennotiert sind und für die Vorschriften zur Arbeitnehmermitbestimmung zu beachten haben. Schließlich fehlt eine Quote für den Vorstand, während sie für den Aufsichtsrat verbindlich ist.

Grundsätzlich beanspruchen die Mitgliedstaaten jedoch im Rahmen des Art. 157 Abs. 4 AEUV ${ }^{168}$ ebenso wie bei der Beschränkung der Niederlassungsfreiheit die politische Einschätzungsprärogative für die Wahl des geeigneten Mittels für sich ${ }^{169}$. Diese Freiheit besteht hier aber nur eingeschränkt, da die Bundesrepublik in einem Politikfeld reguliert, das durch Wertvorstellungen der EU-Gründungsverträge überformt ist und in dem eine entsprechende

$163 \operatorname{COM}(2012) 614$ endg.

164 2012/0299(COD), A7-0340/2013.

165 2012/0299(COD), Debatte vom 11.12.2014.

$166 \mathrm{Vgl}$. auch das dazu gehörige Aktionsprogramm http://ec.europa.eu/justice/genderequality/files/women-on-boards_de.pdf.

167 Anders lautete der Beschluss im Rat, der zur Flexibilisierung und zur Erhöhung der Akzeptanz des Entwurfs bei den Mitgliedstaaten Durchsetzungsalternativen zur Quote vorsieht.

168 Krebber, aaO (Fn. 145), Art. 157 AEUV Rdn. 77.

169 Vgl. statt vieler EuGH, Urt. v. 12.9.2006, Rs. C-196/04 - Cadbury Schweppes, Rdn. 52, wo die Geeignetheit der Maßnahme gar nicht gesondert geprüft wird. 
Regelungskompetenz der Union besteht (Art. 157 Abs. 3 AEUV). Unabhängig davon findet die mitgliedstaatliche Regelungsautonomie jedenfalls im allgemeinen Kohärenzgebot des Art. 7 AEUV ihre Grenzen ${ }^{170}$. Unter dem Kohärenzgebot steht den Mitgliedstaaten zwar insbesondere in kulturell und sittlich empfindlichen Gebieten die Wahl des Schutzniveaus und der Mittel frei ${ }^{171}$. Die Union ist aber befugt zu überprüfen, ob eine Maßnahme aus $e x$ ante-Sicht zielführend sein kann ${ }^{172}$. Übertragen auf den konkreten Fall stellt das Kohärenzgebot also die Frage, ob das nur selektive Ansetzen bei den Aufsichtsräten mitbestimmter und börsennotierter Kapitalgesellschaften das hinter der Quote stehende gesellschaftspolitische Ziel der Geschlechterparität im Wirtschafts- und Arbeitsleben nicht von vornherein ungeeignet erscheinen lässt.

Ein Blick auf das aktuelle Gesetzgebungsvorhaben der Union entkräftet jedoch einen etwaigen dahingehenden Einwand nachhaltig. Sowohl der Aktionsplan des Europäischen Parlaments als auch der Kommissionsentwurf zur Geschlechterquote lassen nahezu identischen Zuschnitt erkennen wie die deutsche Regelung, da auch hier eine Quote nur für die Leitungsorgane börsennotierter Großunternebmen vorgesehen ist ${ }^{173}$. Dass die Bundesregierung den Kreis der betroffenen Unternehmen noch weiter verengt, indem sie erstens nur mitbestimmte Kapitalgesellschaften erfassen will und zweitens die Wahl der Vorstände aus dem Geltungsbereich der Quote ausklammert, erklärt sich dadurch, dass weder die Arbeitnehmermitbestimmung noch die dualistische Leitungsstruktur von Kapitalgesellschaften in allen EU-Mitgliedstaaten bekannt ist.

\section{Erforderlichkeit und Angemessenheit}

Auch die Erforderlichkeit einer 40\%-Quote in Aufsichtsräten lässt sich mit Blick auf den Richtlinienentwurf der Kommission und seine Rezeption in den Gesetzgebungsorganen der Union ohne weiteres bejahen, da jene ein identisches Vorhaben enthalten ${ }^{174}$. Dass im Rat Vorbehalte gegen die Exklusivität der Quotenregelung als Durchsetzungsmechanismus für die geschlechterparitätische Besetzung von Führungsgremien laut wurden und infolge dessen

170 Frenz, Kohärente und systematische nationale Normgebung, EuR 2012, 344; das deutsche Grundgesetz reflektiert diese Vorgabe durch den Grundsatz widerspruchsfreier Normgebung, dazu BVerfGE 98, 83 - Landesabfallabgaben; BVerfGE 98, 106 Kommunale Verpackungsteuer; Frenz, Das Prinzip widerspruchsfreier Normgebung und seine Folgen, DÖV 1999, 41.

171 Frenz, EuR 2012, 344, 345.

172 Frenz, EuR 2012, 344, $348 \mathrm{f}$.

$173 \operatorname{COM}(2012) 614$ endg., 2.

$174 \operatorname{COM}(2012) 614$ endg. 
entsprechende Härte- und Ausweichklauseln in den Richtlinienentwurf eingefügt wurden ${ }^{175}$, stellt als Spezifikum unionsweiter Rechtssetzung die Primärrechtskonformität des Gesetzes nicht in Frage. Erstens muss ein Legislativakt der Union im Gegensatz zum Gesetz der Bundesregierung die Spezifika diverser unterschiedlicher Gleichstellungspolitiken in 28 Mitgliedstaaten berücksichtigen. Zweitens darf nicht unbeachtet bleiben, dass Kommission und Parlament die Geschlechterquote nicht durch eine Verordnung, sondern durch eine mindestharmonisierende Richtlinie umsetzen möchten ${ }^{176}$. Die Mitgliedstaaten sind daher unionsrechtlich befugt, das Ziel der paritätischen Besetzung durch eine fixe Quotenregelung ohne Härtefallklausel durchzusetzen ${ }^{177}$.

\section{Zusammenfassung in Thesenform}

1. Die Geschlechtermindestquote kann gemäß $\ 17$ Abs. 2 SEAG-Entwurf und $\$ 24$ Abs. 3 SEAG-Entwurf auf die dualistische und monistische SE erstreckt werden. Der deutsche Gesetzgeber hat entgegen anderslautender Stimmen in der Literatur die entsprechende Regelungskompetenz. Sie folgt bei einer SE mit Satzungssitz im Inland aus der Verweisung des Art. 9 Abs. 1 lit. c SE-VO auf das deutsche Recht als sekundäres Personalstatut der SE. Weder das primäre Personalstatut in Gestalt der SE-VO noch die SE-Mitbestimmungsrichtlinie regeln die Quote; sie entfalten auch keine Sperrwirkung in Bezug auf eine Quotenregelung auf Ebene des sekundären Personalstatuts einer SE.

2. Die reguläre Verweisung der Gründungstheorie auf das (ausländische) Gesellschaftsstatut wird durchbrochen über die Sonderanknüpfung von Eingriffsnormen der lex fori, zu denen auch die starre Geschlechterquote nach \$S 96 Abs. 2 AktG-Entwurf, 17 Abs. 2 und 24 Abs. 3 SEAG-Entwurf zu rechnen ist.

3. $\int \mathbb{S} 96$ Abs. 2 AktG-Entwurf und 24 Abs. 3 SEAG-Entwurf sind von einem internationalen Anwendungswillen getragen: Die Quotenregelung soll einen Ordnungsrahmen für in Deutschland tätige Unternehmen bereitstellen. Sie greift weit über den konkreten privatrechtlichen Zusammenhang der Auf-

175 2012/0299(COD), Debatte vom 11.12.2014.

$176 \operatorname{COM}(2012) 614$ endg., 12: „In diesem Vorschlag für eine Mindestharmonisierung werden lediglich gemeinsame Ziele festgelegt. Den Mitgliedstaaten wird ausreichend Handlungsspielraum bei der Entscheidung gelassen, wie diese Ziele auf nationaler Ebene unter Berücksichtigung der jeweiligen Rahmenbedingungen auf nationaler, regionaler oder lokaler Ebene, insbesondere des Gesellschaftsrechts sowie der Regeln und Verfahren für die Berufung von Mitgliedern der Leitungsorgane von Unternehmen, am besten erreicht werden sollten."

177 Für die verfassungsrechtliche Bedenklichkeit der fixen Quotenregelung aber WINDTHORst, Stellungnahme, BT-Drucks. 18/3784, 5 ff. 
sichtsratswahl hinaus und ist orts- im Gegensatz zu (gesellschafts-)statutsbezogen.

4. Nur die 30\%-Quotenvorgabe als solche ( $\mathbb{S} 96$ Abs. 2 Satz 1 AktG-Entwurf und $\$ 24$ Abs. 3 Satz 1 SEAG-Entwurf) findet qua Sonderanknüpfung auf Auslandsgesellschaften Anwendung, dagegen weder das Implementierungsregime (Modus der Bestellung der Aufsichtsratsmitglieder) noch die Folgen einer quotenwidrigen Besetzung (Nichtigkeitsfolge, $\$ 96$ Abs. 2 Satz 6 AktGEntwurf)

5. Die kollisionsrechtliche These, der zufolge sich die Quotenregelung auch auf ausländische Gesellschaften erstreckt, und die sachrechtliche These, nach der das persönliche Tatbestandsmerkmal „AG“ durch ausländische Rechtsformen substituiert werden kann, sind bei normlogischer Betrachtung gleichwertig. Als weitere Voraussetzung einer Sonderanknüpfung der Quotenvorgabe müssen deshalb die tatbestandlich ersetzte deutsche Gesellschaftsform und die im Wege der überbrückenden Substitution eingesetzte ausländische Gesellschaftsform funktionell gleichwertig sein. Die Quotenregelung erstreckt sich daher im Ergebnis nur auf ausländische börsennotierte Publikumsgesellschaften mit einer Regelbeschäftigung von mehr als 2000 Arbeitnehmern.

6. Eine solche expansive Form der Substitution muss demselben Rechtfertigungsdruck unterworfen werden, dem jeder Eingriff in die kollisionsrechtliche bestimmte lex causae standzuhalten hat. Somit ist auch unter dem sachrechtlichen Aspekt der expansiven Substitution eine enge Verbindung in Form eines hinreichenden Inlandsbezugs zu verlangen. Dieser liegt dann vor, wenn die Auslandsgesellschaft ihren Verwaltungssitz in Deutschland hat und dort regelmäßig mehr als 2000 Arbeitnehmer beschäftigt.

7. Die Erstreckung der Quotenregelung auf derartige Auslandsgesellschaften ist europarechtskonform. Wenn man sie nicht schon als Keck-Ausnahme ansehen will, stellt sie zwar eine Beschränkung der Niederlassungsfreiheit dar; diese lässt sich jedoch mit zwingenden Gründen des Allgemeinwohls rechtfertigen. 